\title{
Deep Neural Network-based Enhancement for Image and Video Streaming Systems: A Survey and Future Directions
}

\author{
ROYSON LEE, University of Cambridge, UK \\ STYLIANOS I. VENIERIS, Samsung AI Center, Cambridge \\ NICHOLAS D. LANE, Samsung AI Center, Cambridge \& University of Cambridge, UK
}

\begin{abstract}
Internet-enabled smartphones and ultra-wide displays are transforming a variety of visual apps spanning from on-demand movies and $360^{\circ}$ videos to video-conferencing and live streaming. However, robustly delivering visual content under fluctuating networking conditions on devices of diverse capabilities remains an open problem. In recent years, advances in the field of deep learning on tasks such as super-resolution and image enhancement have led to unprecedented performance in generating high-quality images from low-quality ones, a process we refer to as neural enhancement. In this article, we survey state-of-the-art content delivery systems that employ neural enhancement as a key component in achieving both fast response time and high visual quality. We first present the components and architecture of existing content delivery systems, highlighting their challenges and motivating the use of neural enhancement models as a countermeasure. We then cover the deployment challenges of these models and analyze existing systems and their design decisions in efficiently overcoming these technical challenges. Additionally, we underline the key trends and common approaches across systems that target diverse use-cases. Finally, we present promising future directions based on the latest insights from deep learning research to further boost the quality of experience of content delivery systems.
\end{abstract}

CCS Concepts: • General and reference $\rightarrow$ Surveys and overviews; $\bullet$ Computing methodologies $\rightarrow$ Computer vision tasks; Neural networks; Distributed computing methodologies;

Additional Key Words and Phrases: Deep learning, content delivery networks, distributed systems

ACM Reference format:

Royson Lee, Stylianos I. Venieris, and Nicholas D. Lane. 2021. Deep Neural Network-based Enhancement for Image and Video Streaming Systems: A Survey and Future Directions. ACM Comput. Surv. 54, 8, Article 169 (October 2021), 30 pages.

https://doi.org/10.1145/3469094

\section{INTRODUCTION}

Internet content delivery has seen a tremendous growth over the past few years. Specifically, video traffic is estimated to account for $82 \%$ of global Internet traffic by 2022 -up from $75 \%$ in

Royson Lee and Stylianos I. Venieris contributed equally to this research.

Authors' addresses: R. Lee, University of Cambridge, The Old Schools, Trinity Lane, CB2 1TN, Cambridge, UK; email: dsrl2@cam.ac.uk; S. I. Venieris, Samsung AI Center, 50/60 Station Road, CB1 2JH, Cambridge, UK; email: s.venieris@ samsung.com; N. D. Lane, Samsung AI Center, 50/60 Station Road, CB1 2JH, Cambridge, UK \& University of Cambridge, The Old Schools, Trinity Lane, CB2 1TN, Cambridge, UK; email: nic.lane@samsung.com.

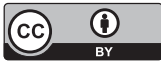

This work is licensed under a Creative Commons Attribution International 4.0 License.

(C) 2021 Copyright held by the owner/author(s).

0360-0300/2021/10-ART169 \$15.00

https://doi.org/10.1145/3469094 
$2017[23,124]$. This growth is attributed to not only the rapid increase of Internet-enabled devices, but also the support for higher-resolution content. For instance, an estimated $66 \%$ of TV sets will support ultra-high-definition (4K) videos by 2023 as compared to $33 \%$ in 2018 [22]. Most importantly, content traffic such as live streaming, video-conferencing, video surveillance, and both short- and long-form video-on-demand, are expected to rise very quickly. To meet these demands, a new class of distributed systems has emerged. Such systems span from content delivery systems (CDS) $[114,166]$ that aim to maximize the user satisfaction and quality of experience (QoE), to video analytics frameworks that provide support for augmented and virtual reality apps by co-optimizing latency and accuracy [36, 152].

One of the primary challenges of distributed systems for content delivery is their reliance on networking conditions. Currently, the quality of the communication channel between client and server plays a key role in meeting the application-level performance needs due to the significant amount of transferred data and the tight latency targets. Nevertheless, in real-life mobile networks, the communication speed fluctuates and poor network conditions lead to excessive response times, dropped frames, or video stalling, which rapidly degrade the user experience. This phenomenon is further amplified by the increasing number of users that compete for the same pool of network resources.

For many years, bitrate adaptation has been the dominant approach for counteracting the networking dynamicity and the main driver behind adaptive video streaming [11], with already deployed solutions by industry content providers such as iQiyi [140], Netflix [62], and YouTube [103]. Under this scheme, each video is broken down into segments that are encoded using multiple bitrates. Upon client's request, the segments are streamed over HTTP, with the client selecting the bitrate in a per-segment manner. Adaptive bitrate (ABR) algorithms typically consider either the connection speed $[71,100,172]$ or the playback buffer state $[63,137]$, to dynamically select the bitrate of each video segment. Although ABR technology has achieved significant gains in sustaining high QoE across diverse settings, existing approaches still leave substantial portion of the available bandwidth underutilized $[61,103]$ either due to the ABR algorithm's suboptimality or due to the service provider's policy of minimizing bandwidth usage. With ABR leaving substantial room for further optimization, there is an emerging need for novel techniques to further boost the performance of content delivery systems and ensure high QoE across various visual content delivery applications, network conditions, and device capabilities.

One of these techniques includes the use of newly emerging technologies such as telepresence [170]. For instance, the recently released Nvidia Maxine [120] can be used to drastically reduce the network load by allowing each user to only transmit at least one key frame, which is used to initialize the model at the receiver's end. During video-conferencing, facial landmarks, which use considerably less bandwidth than frames, are exchanged and used by their respective models to synthesize the video and mimic the other user's facial expressions, hence resulting in a fast and accurate video-conferencing experience even under extremely low-bandwidth settings. However, the usage of such techniques is limited and solely for video-conferencing, as working in dynamic environments or with multiple users is still an unsolved challenge.

Another recent key method that enables tackling this challenge in general is neural enhancement through super-resolution (SR) and image enhancement models. These models are capable of processing a low-resolution or low-quality image and generating a high-quality output. With the unprecedented performance of convolutional neural networks (CNNs), content delivery systems have begun integrating neural enhancement models as a core component. The primary paradigm of using neural enhancement models in content delivery systems comprises the transmission of compact low-resolution or low-quality content, often along with the associated neural model, followed by its subsequent quality enhancement on the receiver side through a 
Table 1. Overview of Visual Content Delivery Systems

\begin{tabular}{llr}
\hline System & \multicolumn{1}{c}{ Task } & \multicolumn{1}{c}{ Year } \\
\hline MobiSR [92] & On-demand image delivery & October 2019 \\
Yeo et al. [165] & On-demand video delivery & November 2017 \\
NAS [166] & On-demand video delivery & October 2018 \\
NEMO [164] & On-demand video delivery & September 2020 \\
PARSEC [28] & $360^{\circ}$ video delivery & August 2020 \\
Supremo [167] & On-demand image delivery & September 2020 \\
CloudSeg [152] & Video segmentation & July 2019 \\
Dejavu [54] & Video-conferencing & February 2019 \\
LiveNAS [79] & Live streaming & July 2020 \\
SplitSR [108] & Digital zoom & March 2021 \\
\hline
\end{tabular}

enhance-capable model [165]. In this manner, the transfer load is minimized, drastically reducing the network footprint and the corresponding bandwidth requirements, with the visual quality recovered on the client side.

Despite their benefits, integrating state-of-the-art neural enhancement models into visual content delivery systems poses significant challenges. First, these models, especially SR models, have excessive computational demands that are measured up to hundreds of TFLOPs per frame to achieve an upscaling of up to $4 \mathrm{~K}$ or $8 \mathrm{~K}$. With client platforms typically involving devices with strict resource and battery constraints $[6,85,146]$, clients are still struggling to execute neural enhancement models on-device while meeting the target quality [92]. This fact is aggravated by the stringent latency and throughput requirements that are imposed to sustain high QoE. Finally, enabling the deployment of such systems requires overcoming unique technical challenges stemming from the diversity of use-cases, spanning from on-demand video streaming [166] to videoconferencing [54].

In this article, we provide a timely and up-to-date overview of the growing area of visual content delivery systems that employ neural enhancement. Through this survey, we aim to equip researchers new to the field with a comprehensive grounding of next-generation content delivery system design, revealing how neural enhancement techniques can lead to greater performance than status quo methods. More specifically, we make the following novel contributions:

- We motivate neural enhancement for CDS by describing the architecture, major components, performance metrics, and open challenges of conventional content delivery systems.

- We survey the state-of-the-art existing systems that utilize neural enhancement (Table 1) across diverse content delivery applications including on-demand video and image services, visual analytics, video-conferencing, live streaming, and $360^{\circ}$ videos. We detail their neural model design and system optimization strategies, assessing their strengths and limitations.

- Drawing from the latest progress by the computer vision community, we propose several promising future directions for future research and describe how they can be integrated to value-add existing and inspire future content delivery systems.

\section{VISUAL CONTENT DELIVERY SYSTEMS}

Content delivery systems are systems that aim to deliver image or video content to the users with minimal latency and high visual quality, while supporting a diverse set of client platforms. Across the years, several different aspects of CDS have been studied, from optimized networking $[81,101]$ 
and system design $[10,26,33,43,114]$ to user behavior $[98,169]$, cost minimization [56, 94], and performance evaluation [2, 47, 97, 99]. Common underlying challenges for sustaining high performance and meeting the agreed-upon QoS are the client device heterogeneity-from powerful desktops to diverse mobile devices [157]-and the reliance on networking conditions. In this section, we present (1) the common performance metrics, (2) the typical architecture of CDS, and (3) the adaptive bitrate methods that are conventionally used to counteract bandwidth fluctuations. Then, we introduce (4) the recent technique of neural enhancement in terms of its principle of operation and the associated technical challenges.

\subsection{Performance Evaluation Metrics}

The performance evaluation of CDS is complex, comprising multiple metrics with often competing dynamics. The primary metrics of interest include essential attributes such as visual quality, frame rate, response time, rebuffering time, accuracy, and quality of experience. Based on the characteristics of the target task, the design of CDS often prioritizes a subset of metrics and aims to reach a task-specific balance among them. Therefore, measuring and reporting all these criteria across diverse scenarios plays an important role in determining the strategic tradeoffs made by CDS.

Visual Quality. Traditionally, the bitrate dictates the maximum resolution of the content without rebuffering; the higher the bitrate, the higher the supported resolution and thus the better the visual quality. With the introduction of neural enhancement models in CDS, the maximum resolution is determined by both the bitrate and the amount of computational resources available to execute these models. Additionally, the visual quality of the content is influenced not only by its maximum resolution, but also by the performance of the model used to enhance the content, i.e., the model's enhancement capability. The dependency on the bitrate for visual quality is therefore relaxed and the extent of this dependency is determined by the available compute, upscale factor, and degree of compression. In other words, given sufficient computational resources, the impact on the content's visual quality is shifted towards the performance of the neural model. For instance, higher upscale factors and more computational resources enable the streaming of high-definition resolution at lower bitrates and thus rely on the performance of the model for high visual quality.

The visual performance of a neural enhancement model is often measured using either (i) a distortion-based metric, such as Peak Signal-to-Noise Ratio (PSNR) [44] or Structural Similarity Index Measure (SSIM) [155], or (ii) a perceptual-based metric, such as Naturalness Image Quality Evaluator (NIQE) [117] or Learned Perceptual Image Patch Similarity (LPIPS) [174]. Although optimizing using a perceptual-based metric will lead to more natural-looking images, existing CDS adopt distortion-based metrics, which aim to maximize image fidelity. These metrics are resolution-agnostic and the visual quality is determined solely by the absolute pixel-to-pixel error or their inter-dependencies between the original frame and its reconstructed version at the receiver. To accommodate these model performance metrics into traditional QoE metrics that utilized solely the bitrates, some existing neural enhancement-based CDS directly map these model performance metrics into bitrates as detailed below.

Accuracy. In video analytics use-cases [36,152], such as security surveillance, traffic monitoring, and face recognition, the most commonly used performance metric is accuracy, which captures the proportion of correct classifications over the input samples processed upon deployment. Accuracy is reported as percentage for classification tasks or in the range $0-1$ for the Intersection-overUnion (IoU) metric of semantic segmentation tasks [110].

Response Time. Latency, or response time, is the primary performance indicator in latencysensitive interactive applications, such as virtual reality [18, 30, 107], video analytics services [152], 
and video-conferencing [54]. Measured in seconds, response time is the end-to-end time between when an input event occurs (e.g., new frame in the user's real-time video or caller makes a gesture) and when the output is returned to the user's device (e.g., analytics result or enhanced view of caller). In such scenarios, excessive buffering adds a prohibitive latency overhead that degrades the QoE and is often not an option.

Frame Rate. Frame rate is among the primary metrics of interest in throughput-driven applications, such as video-on-demand [7, 90, 164, 166] and live video streaming [79]. Frame rate is measured in frames per second (fps), with the lower bound of real-time frame rate being between 24-30 fps.

Rebuffering. A critical metric for characterizing the user-perceived quality of video applications is rebuffering [118]. This phenomenon occurs when the playback buffer is drained due to the slow transmission of video segments, with the video player stalling and the playback pausing. Rebuffering captures the temporal properties of a video playback, independently of its content, and is typically analyzed with respect to: (1) initial buffering time, the time between the request of a video by the user and the beginning of its playback; (2) mean rebuffering duration, the average time of a rebuffering event; and (3) rebuffering frequency, the occurrence frequency of rebuffering events.

Quality of Service (QoS). Network-level QoS captures the performance of a network connection and its capabilities to provide packet transfer with the agreed-upon requirements [39]. QoS is typically quantified using a set of networking-level metrics, including delay, jitter, packet loss rate, and bandwidth. By monitoring these quantities, service providers can tune network parameters and apply traffic shaping to meet specific service-level agreements (SLAs) and sustain a high QoS. In this endeavor, the expected outcome is that high network-level QoS corresponds to high user satisfaction for the underlying application.

Quality of Experience (QoE). Despite the merits of network-level QoS, the relation between QoS measurements and user satisfaction is not trivial. With a more user-centric perspective, quality of experience (QoE) aims to capture the quality of an application or service from the point of view of the users. QoE is typically assessed either via subjective or objective methods. To directly measure the subjective perceived quality, a group of actual users are asked to provide a quality score over a sequence of videos to obtain a mean opinion score (MOS) [68]. Despite the accuracy of this approach, it relies on significant manpower and has to be conducted offline [5], preventing the automated monitoring of QoE that is required by real-time video applications.

With the subjective approach not deployable, objective methods have emerged. Such approaches consist of continuously collecting network-level QoS measurements together with application performance metrics [118], such as video bitrate, mean rebuffering time, rebuffering frequency, and per-frame spatial quality [122], and using analytical formulas to aggregate them to automatically estimate QoE. Such an approach is adopted by the majority of the covered content delivery systems to track in real-time the achieved QoE and adapt accordingly.

The most commonly used objective QoE metric (Equation(1)) $[32,61,168]$ to date for neural enhancement-based CDS takes into account the bitrate of each video sequence, $R$, and rebuffering measures. In this respect, QoE is generally defined as a linear combination of: the bitrate utility, $q(R)$, that maps bitrate $R$ to video quality; the rebuffering time caused by downloading the $n$th segment, $T_{n}$; the initial buffering time, $T_{s}$; and the smoothness of the selected quality, $q\left(R_{n+1}\right)-$ $q\left(R_{n}\right)$, which captures the variation in visual quality between subsequent segments $(n$ and $n+1)$ 


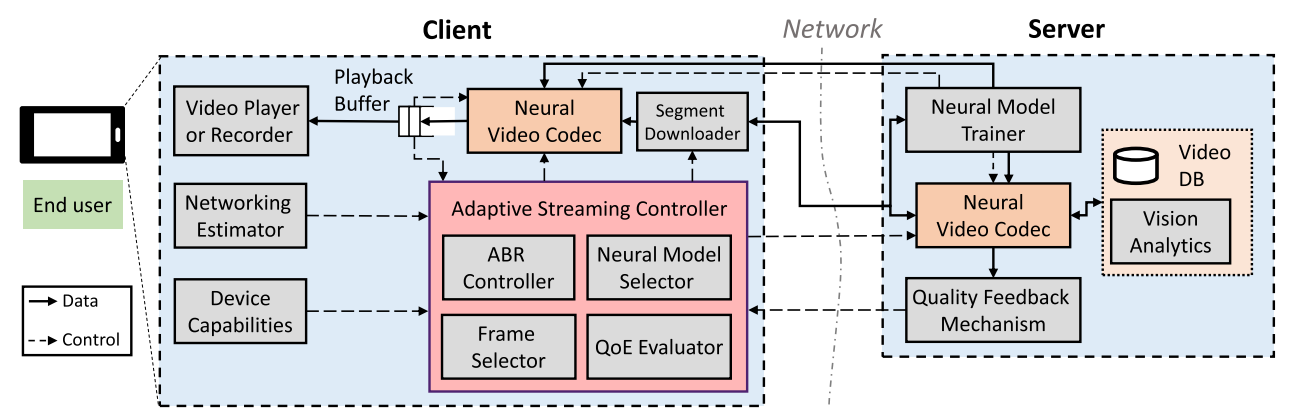

Fig. 1. Architecture of content delivery systems.

in terms of both their number and amplitude.

$$
Q o E=\frac{\alpha \sum_{n=1}^{N} q\left(R_{n}\right)-\beta \sum_{n=1}^{N} T_{n}-\gamma \sum_{n=1}^{N-1}\left|q\left(R_{n+1}\right)-q\left(R_{n}\right)\right|}{N}-\delta T_{s},
$$

where $N$ is the total number of video sequences, and the bitrate utility is modeled as linear [168], $q(R)=R$, logarithmic [137], $q(R)=\log \left(R / R_{\min }\right)$, to diminish improvement at higher bitrates, or fixed [114] to favor higher-definition bitrates. Hyperparameters $\alpha, \beta, \gamma$, and $\delta$ determine the importance of the four components, penalizing the average segment quality, rebuffering delay, smoothness of quality variation, and initial buffering time, respectively.

To take into account the neurally enhanced quality of the videos, a few works $[164,166]$ used an inverse mapping from quality to bitrate through interpolation (e.g., piecewise linear interpolation). Specifically, $\widehat{R}_{n}=V_{n} f^{-1}\left(f\left(m\left(V_{n}\right)\right)\right)$ where $V$ is the video sequence, $m$ is the neural enhancement model and $f$ is the model's performance metric. The QoE is, therefore, determined using the estimated bitrate, $\widehat{R}_{n}$, given the performance of the model instead of the actual bitrate.

Other considerations. In addition to traditional performance measures, other metrics of interest for CDS are the server-provider-side (SP-side) and user-side costs. On the SP side, costs can be quantified based on bandwidth usage, per user or aggregate, or processing time on the SP's servers. Analytically, these can be captured either as additional hard constraints with specific bandwidth budget and computation time limit, or as additional objectives to be optimized, where the bandwidth and server resource usage are minimized. On the user side, cost can be quantified based on the additional energy overhead or the computational and memory resource usage imposed by running neural enhancement on the client device. Similarly to SP-side costs, these can be modeled either as hard constraints or as metrics in an optimization problem that maximizes energy efficiency and minimizes processing time.

\subsection{Architecture}

CDS typically adopt distributed architectures consisting of one or multiple clients and servers. Figure 1 depicts the typical architecture of such a system. Focusing on video-on-demand (VOD) without loss of generality, the operation starts on the client side with the user selecting an online video to watch and the video player app (Video Player) sending a request to the video server. Next, the server fetches the video from its database (Video $D B$ ) and launches a transmission-preparation stage. In modern adaptive video streaming services $[11,138]$, instead of sending one large monolithic video file, the video content is divided into a series of seconds-long segments (e.g., 4 seconds per segment). This approach enables the client's Adaptive Streaming Controller to request different bitrate encoding per segment through the ABR Controller to dynamically adapt to changing environmental conditions [29]. To this end, each video segment is compressed by the server-side Video 
Codec using a predefined encoding scheme and the client-specified bitrate. Finally, the client's Segment Downloader pulls the encoded segments, where they are decoded by the client-side Video Codec and passed to the Playback Buffer to be concatenated and eventually played. As discussed in Section 4, depending on the target application and adopted design decisions, CDS may optionally integrate additional components such as a Frame Selector, Model Selector and/or QoE Evaluator on the client side, and a Quality Feedback Mechanism and/or a Vision Analytics backend on the server side. All processing stages are typically pipelined to enable streaming operations and a higher attainable throughput.

For such systems to meet the performance targets, the communication channel between client and server has to sustain high bandwidth throughout the streaming process. This constitutes a strong assumption that breaks for mobile clients where the connectivity conditions vary continuously. Hence, additional techniques, such as adaptive bitrate and neural enhancement, have been introduced that enable the dynamic adaptation to the instability of the channel.

\subsection{Bitrate Adaptation}

To remedy the dependence of content delivery systems on network conditions, ABR algorithms have emerged $[11,61,63,71,100,114,137,168,172]$. The majority of the existing bitrate adaptation methods reside on the client side, complying with the Dynamic Adaptive Streaming over HTTP (DASH) standard [138] and enabling large-scale deployment by applying only client-side modifications [11]. Under this scheme, the client device first monitors its instantaneous bandwidth (Networking Estimator in Figure 1) to assess the current network state [71, 100], or the occupancy of its Playback Buffer [63, 137], or both [4,136, 168]. Next, the ABR Controller tunes accordingly the per-segment bitrate that it requests from the server and configures the client's codec to decode at the selected rate. On the remote side, the server encodes each video segment with the specified bitrate and responds to the Segment Downloader's pull request. Overall, ABR techniques function as a way to control the network footprint at runtime and thus help to minimize rebuffering. Although ABR has been significantly improved through deep learning-based algorithms $[41,60,61,114,172]$ to better perform under unexpected settings, it often fails in scarce network conditions as it relies solely on network resources.

\subsection{Neural Enhancement}

Neural enhancement aims to restore and recover the quality/resolution of visual input. As this problem is inherently ill-posed (i.e., many high-resolution solutions can be downsampled to the same low-resolution image), most works enforce a strong prior to mitigate its ill-posed nature. To this end, most state-of-the-art approaches utilize CNNs to capture the prior, as it results in superior visual performance. These methods train a model to either map a low- to a high-quality image using exemplar pairs $[34,78,104]$ or exploit the internal recurring statistics of the image to enhance/upscale it [130].

The primary paradigm of using neural enhancement models in content delivery systems comprises the transmission of compact low-resolution/low-quality content followed by its subsequent enhancement on the receiver side through the enhance-capable models $[165,166]$. In this manner, the transfer load is tunable by means of the upscaling factor (for SR) and the degree of compression, controlling the system's network footprint and the associated bandwidth requirements. Therefore, neural enhancement opens up a new dimension in the design space by introducing a tradeoff between computational and network resources, effectively overcoming existing systems' sole reliance on network resources. To this end, existing systems may choose to independently optimize the utilization of these neural enhancement models [79, 92, 167] or integrate them within existing ABR algorithms [28, 164, 166]. 
Table 2. Comparison of Neural Enhancement Models used in CDS (Section 4) for Commonly Used Scaling Factors on Standard Benchmark Datasets

\begin{tabular}{|c|c|c|c|c|c|c|c|c|}
\hline Scale & Model & & $\begin{array}{c}\text { Params } \\
(\mathrm{K})\end{array}$ & $\begin{array}{l}\text { Mult-Adds } \\
(\mathrm{G})\end{array}$ & $\begin{array}{c}\text { Set5 [12] } \\
\text { PSNR/SSIM }\end{array}$ & $\begin{array}{l}\text { Set14 [162] } \\
\text { PSNR/SSIM }\end{array}$ & $\begin{array}{l}\text { B100 [115] } \\
\text { PSNR/SSIM }\end{array}$ & $\begin{array}{c}\text { Urban100 [59] } \\
\text { PSNR/SSIM }\end{array}$ \\
\hline \multirow{6}{*}{$2 \times$} & VDSR & [78] & 665 & 612.6 & $37.53 / 0.9587$ & $33.03 / 0.9124$ & $31.90 / 0.8960$ & $30.76 / 0.9140$ \\
\hline & CARN & [3] & 1,592 & 222.8 & $37.76 / 0.9590$ & $33.52 / 0.9166$ & $32.09 / 0.8978$ & $31.92 / 0.9256$ \\
\hline & IDN & [64] & 552 & 202.8 & $37.83 / 0.9600$ & $33.30 / 0.9148$ & $32.08 / 0.8985$ & $31.27 / 0.9196$ \\
\hline & EDSR & [104] & 40,711 & $9,384.7$ & $38.11 / 0.9601$ & $33.92 / 0.9195$ & $32.32 / 0.9013$ & $32.93 / 0.9351$ \\
\hline & MDSR & [104] & 7,953 & $1,501.5$ & $38.11 / 0.9602$ & $33.85 / 0.9198$ & $32.29 / 0.9007$ & $32.84 / 0.9347$ \\
\hline & RCAN & [175] & 15,444 & $3,526.8$ & $38.27 / 0.9614$ & $34.12 / 0.9216$ & $32.41 / 0.9027$ & $33.34 / 0.9384$ \\
\hline \multirow{6}{*}{$4 \times$} & VDSR & [78] & 665 & 612.6 & $31.35 / 0.8838$ & $28.01 / 0.7674$ & $27.29 / 0.7251$ & $25.18 / 0.7524$ \\
\hline & CARN & [3] & 1,592 & 90.9 & $32.13 / 0.8937$ & $28.60 / 0.7806$ & $27.58 / 0.7349$ & $26.07 / 0.7837$ \\
\hline & IDN & [64] & 552 & 89.0 & $31.82 / 0.890$ & $28.25 / 0.7730$ & $27.41 / 0.7297$ & $25.41 / 0.7632$ \\
\hline & EDSR & [104] & 43,070 & $2,894.5$ & $32.46 / 0.8968$ & $28.80 / 0.7876$ & $27.71 / 0.7420$ & $26.64 / 0.8033$ \\
\hline & MDSR & [104] & 7,953 & 410.6 & $32.50 / 0.8973$ & $28.72 / 0.7857$ & $27.72 / 0.7418$ & $26.67 / 0.8041$ \\
\hline & RCAN & [175] & 15,592 & 916.9 & $32.63 / 0.9002$ & $28.87 / 0.7889$ & $27.77 / 0.7436$ & $26.82 / 0.8087$ \\
\hline
\end{tabular}

\section{NEURAL ENHANCEMENT AND ITS DEPLOYMENT CHALLENGES}

So far, a wide range of neural enhancement models and techniques have been integrated in content delivery systems. Although the computer vision field of neural enhancement includes both SR and image enhancement neural networks, CDS generally adopt SR models as reducing the spatial dimensions is more effective at reducing network usage. An exception to this approach is a subset of systems [54] that repurpose SR models for image enhancement by feeding a low-quality inputwhich is of the same resolution as its output-to the SR model. Moreover, existing systems adopt CNNs for supervised single-image SR, due to (1) its efficiency as opposed to multi-frame superresolution $[141,143,148]$ and (2) the availability of the degradation operation as compared to blind unsupervised or self-supervised methods $[9,46,130]$. In this section, we provide an overview of SR models (Table 2) used in existing content delivery systems (Section 4) and discuss their unique deployment challenges.

\subsection{Neural Enhancement Models Used in Content Delivery Systems}

After the first proposed super-resolution CNN [34] that only used three layers, VDSR [78] was the first SR model that used a significantly deep (20-layer) convolutional network to obtain substantial performance gains and is one of the first SR models adopted for on-demand video streaming [165]. The model takes in an interpolated low-resolution input and passes it through 20 convolutional layers, before merging through addition the result with a final residual connection from the input. Each convolutional layer has 64 feature maps followed by a ReLU [119] activation function. Although VDSR is relatively lightweight in terms of workload and memory requirements, Yeo et al. [165] showed that real-time upscaling to $720 \mathrm{p}$ is only possible when the number of layers and feature maps are dropped by half and targeting a powerful desktop GPU, highlighting the computational challenges that will be discussed in Section 3.2.

EDSR [104], winner of the NTIRE 2017 Super-Resolution Challenge [144], and its multi-scale variant, MDSR, are both heavily adopted in various content delivery systems such as NAS, LiveNAS, PARSEC, Dejavu, LiveNAS, and NEMO. MDSR, in particular, extends EDSR by supporting multiple scales and consists of three stages: (1) a front-end feature extraction stage with separate feature extractors per scale, (2) a number of shared intermediate layers, and (3) independent upsampling layers for each scale. Both models adopt residual blocks from ResNet [49], without the batch normalization [67] layers, as their building block. Specifically, the authors showed empirically that 
the use of batch normalization [67] led to performance degradation in SR, a phenomenon that was further studied in Reference [151], leading to the abandonment of batch normalization layers in subsequent SR model designs. Unlike VDSR, EDSR and MDSR employ a variety of computeefficient techniques to reduce their computational complexity and memory footprint. For instance, they take in a low-resolution input and upscale it only at the end [35] using the more efficient pixel shuffle [129] module as opposed to the more costly deconvolution.

Nevertheless, EDSR and MDSR are still considerably heavier than VDSR, with 43 million and 8 million parameters for EDSR and MDSR, respectively, as compared to 0.6 million parameters for VDSR. As a result, CDS often introduce further optimization strategies to alleviate the excessive workload, allow scalability based on the client's capabilities, and achieve the desired performance-latency tradeoff. Such techniques include the generation of multiple single-scale variants of EDSR/MDSR with fewer layers and feature maps to support heterogeneous clients with varying computational capabilities [164]. Apart from multiple CNN configurations, earlyexit strategies $[57,87,142,161]$ are often utilized to allow the client to adapt to their available resources and execute a partially downloaded model [166]. Other methods to speed up execution include training on the luminance channel as opposed to the RGB channels, deploying patch selection to selectively upscale patches through the models [54], and parallelizing execution across multiple GPUs for higher-end clients [79].

Zhang et al. [175] proposed RCAN, a model that achieves better performance at a lower cost as compared to EDSR and is adopted in MobiSR [92] and SplitSR [108]. RCAN achieves this through channel attention blocks and a residual-in-residual structure as its building block. Specifically, instead of designing residual blocks like in EDSR [104], RCAN consists of residual blocks within residual blocks, named residual groups. Each residual block in each group is followed by channel attention blocks, allowing the model to focus on the more informative components of the image. Similar to EDSR and MDSR, RCAN is computationally heavy and requires smaller variants for it to be deployable. For instance, apart from manually tuning the number of layers and feature maps, MobiSR employed a wide range of tensor decomposition and compression techniques to approximate convolutions in RCAN. Similarly, SplitSR substitutes the standard convolutions with channel splitting operations and tunes the depth of the RCAN to optimize for either quality or latency.

Towards efficiency, Ahn et al. [3] proposed CARN, which utilizes concatenated skip connections [58] in a block named cascading block, which consists of residual blocks and convolutions. The authors showed that adding concatenated skip connections at both a block-wise and layerwise level allowed CARN to be more accurate and efficient compared to VDSR [78]. To further speed up latency, IDN [64] proposed channel splitting [112], thereby only processing a subset of its feature maps for some convolutions in each block. Moreover, IDN utilizes group convolutions [84] that can be executed in parallel. Both CARN and IDN are computationally efficient SR models that are able to run in real-time on a high-end desktop GPU and have thus been adopted by cloud-based solutions such as CloudSeg [152] and Supremo [167], respectively.

Apart from the existing SR models and techniques that have already been adopted in content delivery systems, we discuss other works from the SR literature that can be utilized to further support and optimize the real-world deployment of CDS in Section 5. We refer the reader to existing surveys $[105,156,163]$ for a more comprehensive discussion on deep learning-based SR from a computer vision perspective.

\subsection{Challenges of Neural Enhancement}

Despite their advantages, deep neural enhancement models pose a set of critical challenges. First, neural enhancement CNNs are extremely expensive in terms of both computational and memory 
burden. Key factors behind the resource-intensive nature of these models are the large spatial dimensions of feature maps throughout the model's layers together with the excessive number of memory accesses in memory-bound upscaling operations, such as Pixel Shuffle [129]. In this respect, super-resolution CNNs are orders of magnitude larger than image discriminative models, with TFLOP-scale workloads compared to the tens of GFLOPs for classification models [6]. Similarly, the workload of efficiency-optimized SR models [35, 91] are measured in GFLOPs, whereas their image classification counterparts [52] are measured in MFLOPs [6]. A common approach to significantly reduce the peak runtime memory footprint is to split the image into patches that are processed sequentially, with the partial results stitched together to produce the final upscaled image. Nevertheless, the computational cost is still a big challenge for real-time applications, especially when targeting mobile platforms $[28,65,92]$ where the latency per frame spans from hundreds of milliseconds up to seconds depending on the target image resolution.

Furthermore, models trained on standard datasets that aim to generalize across all videos/images result in outputs of varying performance upon deployment and often fail catastrophically on unexpected inputs $[92,165]$. However, tailoring a CNN towards a specific video/image helps to mitigate this drop in performance at the cost of additional training per video/image [53, 74, 128]. This is typically achieved by first pretraining a model on standard datasets and then producing multiple specialized models by fine-tuning different model variants through additional training iterations either per video category [165], per video [164, 166], per video segment [28], per video-conference caller [54] or on a live video stream [79]. In this respect, a generalization-specialization tradeoff is exposed that system designers need to decide how to control based on the target use-case.

\section{THE LANDSCAPE OF CNN-DRIVEN VISUAL CONTENT DELIVERY}

Despite their deployment barriers, several recent frameworks have incorporated neural enhancement methods into their pipelines and introduced novel techniques for overcoming their challenges. In this context, we survey the state-of-the-art visual content delivery systems that leverage neural enhancement models, taxonomizing them based on the type of content (on-demand or live, video or image), and provide an analysis of how they counteract a the excessive computational requirements and $\mathrm{b}$ the performance variability across different content.

\subsection{On-demand Content Delivery Systems}

4.1.1 On-demand Image Delivery. Image delivery systems consume significantly fewer network resources than their video counterparts, therefore requiring considerably less bandwidth. Nevertheless, apart from reducing the load in a shared communication channel, these systems can help users with a limited mobile data plan to save data. For instance, these systems can be deployed in data-saving mobile app alternatives, such as Facebook Lite and Messenger Lite. In this scenario, instead of disabling the downloading of content when the user is not on Wi-Fi, visual content can be downloaded in low resolution to save data and then be locally enhanced to high quality. In this manner, users can continue to scroll through their feed or send picture messages at ease. This applies to any image-centric application including news apps, dating apps, gallery apps, and many others.

Additionally, as chipsets on commodity devices are gradually getting more powerful $[6,65]$, this enables many of these applications to run fully on-device, avoiding the latency and privacy issues of cloud or edge offloading. In this direction, Lee et al. [92] proposed Mobi SR (Figure 2(a)), a system that capitalizes over the heterogeneous compute engines of modern smartphones, e.g., CPU, GPU, and NPU, through a model selection mechanism to deliver rapid image super-resolution.

As a first step, MobiSR derives two model variants by applying a wide range of compression techniques on a user-provided reference model; the authors use a smaller variant of RCAN [175]. These 
compression techniques mainly consist of low-rank tensor decompositions, such as depthwise separable convolutions [131], and other efficient model designs, such as channel splitting [112], that have been successful in high-level vision tasks. The resulting Pareto-optimal models are then assigned to the different available compute engines and a hardware-aware scheduler (Difficulty Evaluation Unit (DEU)) is deployed during inference to rapidly process the patches of each input image. Specifically, the DEU processes hard-to-upscale patches using a more compact model $\left(m_{1}\right)$ while feeding the easier patches to a larger model $\left(m_{2}\right)$ to obtain higher quality, leveraging on an insight that both large and small models perform similarly on hard-to-upscale patches, with difficulty quantified using the total-variation metric [126]. Hence, the image quality is maximized while meeting the applications' latency constraints (challenge a). With respect to challenge b MobiSR is optimized to achieve higher overall performance through a generic model and does not employ model specialization.

MobiSR, however, requires scheduling using multiple processors to leverage its benefits, and may not be suitable in certain deployment scenarios. In this regard, Liu et al. [108] proposed SplitSR (Figure 2(f)), a system that focuses on optimizing either quality or latency on a single CPU and, thus, making it accessible to a wider range of smartphones and computational settings.Similar to MobiSR, SplitSR mitigates challenge a through an efficient variant of RCAN [175]. The proposed model, however, emphasizes the use of channel splitting operations, instead of depthwise separable convolutions, and tuning the model's depth to reduce the DNN workload. The final DNN model is optimized for either quality or latency. Furthermore, SplitSR extends the TVM deep learning compiler [20] to support operations required in their model and generate highly optimized implementations for mobile CPUs. Finally, although the models in Spli tSR achieve higher image fidelity when compared to the models proposed in MobiSR, both frameworks do not specifically handle the variability in upscaling quality across images (challenge (b). Despite being the state-of-the-art for image-centric on-device use-cases, the processing rates achieved by both MobiSR and SplitSR are still below 24-30 fps and hence not yet suitable for real-time video applications.

4.1.2 On-demand Video Streaming. Video-on-demand (VOD) services allow users to watch content at their suitable time from any Internet-enabled device. The user selects a video that is then fetched and streamed by a video server to the user device. With the majority of VOD services being interactive, on-demand video streaming systems have to yield low response time and minimal rebuffering while not compromising visual quality to maximize the QoE. In achieving these targets, the bottleneck lies in the link between the video server and the client with the bandwidth of the connection directly affecting the end performance.

Yeo et al. [165] presented one of the first works that employed neural enhancement to overcome this limitation and offered a way to utilize the clients' computational power. Specifically, the authors first proposed grouping videos into clusters according to their category (basketball, athletics, etc.) through a clustering module. This classification can be performed by either utilizing image classification models or using the video's metadata and predefined categories provided by content platform providers, such as YouTube. A specialized SR model, VDSR [78], is then trained for each cluster, reducing the performance variation (challenge b) as compared to using a single generic model. Besides RGB frames, they also proposed the use of more compact representations such as their edges or the luminance channel, on top of tuning the spatial size of the frames, to further reduce both the bandwidth and computational resource usage. As these alternative representations contain less information than their corresponding RGB frames, the authors utilized Generative Adversarial Network-based (GAN-based) [45] training to learn the distribution of natural textures to synthesize natural-looking frames. Although the authors showed that these compact representations did not work well in practice with the H.264 codec, they were adopted in later works, such as Dejavu [54], to tackle challenge a in different use-cases. 
To handle the excessive computational needs of neural enhancement models (challenge a), the authors constrained their system to work with up to $720 \mathrm{p}$ videos and targeted homogeneous client platforms hosting powerful desktop-grade GPUs. This limitation was subsequently addressed to accommodate clients with heterogeneous computational capabilities in their extended proposed framework-NAS [166].

In NAS [166] (Figure 2(b)), the authors addressed the problem of heterogeneous clients through the use of early-exit SR models of varying sizes and computational workload, allowing each client to select the appropriate model segments (challenge a) based on both their computational capabilities and runtime device load. To this end, they extended previous reinforcement-learning-based (RL-based) ABR algorithms [114] to decide not only the bitrate $(A B R)$, but also the fraction of the SR model (Model Selector) to be transmitted for each video segment.Specifically, the RL-based network is optimized on a modified QoE metric to take into account the enhancement quality of the content, as detailed in Section 2.1, to make the aforementioned decisions. These model segments are, therefore, progressively sent, incrementally updating the model at the client until the full model is delivered. Further mitigating challenge a, the authors based their early-exit SR model on a smaller variant of MDSR [104], which is quantized at 16-bit half-precision floating-point format and executed on a desktop-grade GPU on the client side to hit the real-time requirement. Finally, instead of categorizing videos into coarse clusters as in Reference [165], NAS tackles challenge b by first pre-training a generic SR model and then fine-tuning a specialized model for each video. Overall, as shown in Figure 2(b), the client selects the bitrate $b$ for the $i$ th video segment and the fraction $j$ of the SR model for the particular video and receives the $i$ th low-resolution segment $s_{i}^{v}$ and the associated fraction of the specialized model $m_{j}^{v}$ for video $v$ that are used by the Super-resolution Processor (SRP)-which is part of the Neural Video Codec in Figure 1-to locally produce a high-resolution output.

Although NAS is able to support heterogeneous clients, these clients are assumed to have at least the computational power of a desktop-class GPU. To support lower-end commodity devices, existing on-device SR systems like MobiSR are inadequate at upscaling in real-time. To this end, Yeo et al. [164] proposed NEMO, a framework that leverages frame dependencies using information from the video codec VP9, trading quality degradation for on-device real-time video streaming and reduced energy consumption. Instead of running SR per frame, NEMO applies SR to selected frames called anchor frames, mitigating challenge a), and uses the cached super-resolved anchor frames and the frame dependencies defined in the codec to upscale the remaining non-anchor frames. Specifically, the client runs a SR-integrated codec that refers to a list of anchor frames sent by the server to decide whether to run the model or reuse previously cached super-resolved frames for anchor and non-anchor frames, respectively. For a non-anchor frame, following the VP9 codec, the SR-integrated codec first uses a reference index to select a previously upscaled frame from the Frame Cache. After this step, the provided motion vector is upscaled via bilinear interpolation and motion-compensated before it is used to warp the selected frame. Last, the residual block of the compressed frame is decoded and upscaled via bilinear interpolation before adding to the resulting warped frame.

Although the VP9 codec provides key frames, which have a high-degree of reference from other frames, NEMO deploys its own anchor frame selection to adhere to a given performance threshold. The algorithm first computes the video quality from all possible anchor point sets in a video and then, following a greedy approach, iteratively selects an anchor point that results in the maximum video quality gain until it reaches the quality requirement. Running the quality measurements over all anchor point sets, however, is infeasible. Therefore, to speed up the algorithm, the authors selected the most impactful anchor point in each frame as a proxy, effectively reducing the number 


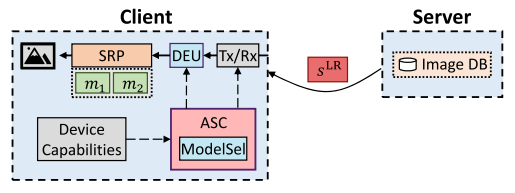

(a)

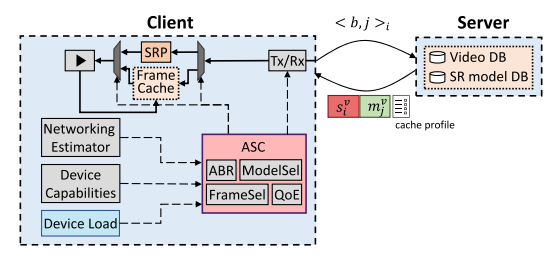

(c)

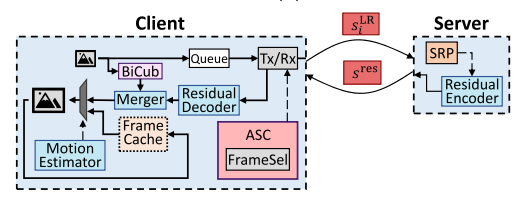

(e)

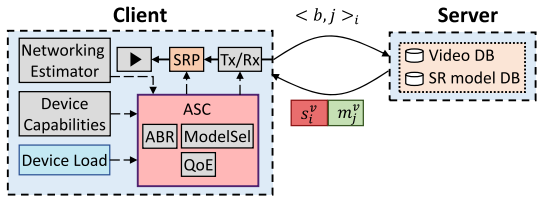

(b)

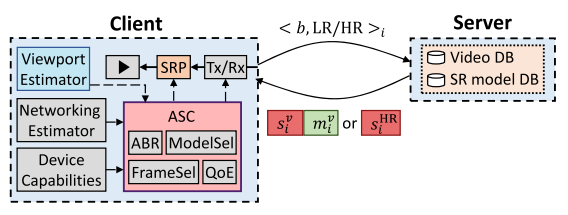

(d)

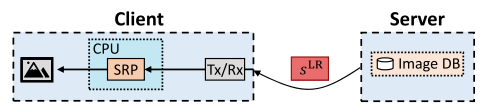

(f)

Fig. 2. Overview of on-demand delivery systems: 2(a)) MobiSR [92], 2(b)) NAS [166], 2(c)) NEMO [164], 2(d)) PARSEC [28], 2(e)) Supremo [167], 2(f)) SplitSR [108]. Cyan blocks indicate novel components introduced by each system.

of anchor point sets to the number of frames. The estimated video quality can thus be quickly calculated as an average of each estimated frame quality, which is computed from a single anchor point, rendering the algorithm computationally feasible.

Following NAS, NEMO alleviates challenges a and b and supports heterogeneous clients by preparing a list of models of various sizes and computational demands, along with a list of their anchor frames generated offline by its anchor frame selection algorithm, for each video and their bitrate version. To guarantee real-time processing, each compute unit in the client processes an anchor and a non-anchor frame once upfront to estimate the overall processing time required for other videos. Given this estimation, the client then selects the available configuration (Model Selector) that maximizes quality and meets the real-time constraint.

4.1.3 $360^{\circ}$ Video Streaming. Compared to regular videos, streaming $360^{\circ}$ videos has significantly elevated bandwidth requirements. To alleviate this, existing systems employ viewport prediction techniques [37] that estimate which part of the video the user will look towards and only download this spatial content. However, accurate viewpoint prediction is still difficult to achieve, exacerbating the problem as missing patches of the current viewpoint need to be fetched at the time of viewing. Therefore, existing solutions [123] send additional patches in the neighborhood of the predicted viewport patches. Although neural enhancement models can be utilized to mitigate this challenge, the larger spatial dimensions of $360^{\circ}$ content further aggravates challenge a and calls for dedicated deployment solutions.

In this context, Dasari et al. [28] proposed a $360^{\circ}$ video streaming framework named PARSEC (Figure 2(d)).Unlike previous works, the authors extended the Adaptive Streaming Controller (Figure 1) logic to decide on the low-resolution (LR) patches to be upscaled (Frame Selector) and the bitrate of high-resolution (HR) patches to be downloaded $(A B R)$ based on (1) the 
networking conditions, (2) the client's computational resources, (3) the viewport prediction (Viewport Estimator), and (4) the quality (PSNR) of both the HR and the upsampled patches. Since the proposed $\mathrm{ABR}$ algorithm is designed to maximize QoE and can thus selectively decide which patches are upscaled or downloaded, challenge $\mathrm{B}$ is mitigated. Moreover, due to the substantial increase in spatial content of $360^{\circ}$ over conventional videos, each manually tuned efficient SR model, similar to that used by NAS, is fine-tuned for each video segment (Model Selector) as opposed to each video.

To overcome challenge a, PARSEC uses an extreme upscaling factor of $\times 64$ on top of the H.265 compression that allows all ultra-LR patches to be transmitted, alleviating the limitations of viewpoint prediction. Additionally, each SR model is varied depending on the targeted quality and the length of the video segment, resulting in shorter inference times for shorter video segments.

4.1.4 Cloud-assisted Content Delivery. In most content delivery scenarios, the client that is requesting the content adaptively allocates the compute needed for enhancement between the server and the client through its ABR algorithm as seen in PARSEC, NAS, and many others. However, for cases in which the ABR algorithm fails, the user's QoE is heavily impacted as the client struggles to handle its workload. To accommodate such cases, computation offloading can be deployed, resulting in a tradeoff between performance and network utilization. Specifically, offloading content to be enhanced offers faster processing, but imposes an extra cost in the bandwidth, along with additional privacy issues, due to the uploading and downloading of low-quality and high-quality content, respectively. Furthermore, this scheme is mainly applicable in use-cases where the lowresolution image already resides in the client device, e.g., pre-downloaded images or videos or zooming in camera-captured images. In such cases, there is no transmission cost to obtain the LR image and hence the additional bandwidth overhead due to offloading might be justified.

To this end, Yi et al. [167] proposed Supremo (Figure 2(e)), a framework that enables real-time mobile SR by selectively offloading computation to the cloud. To mitigate challenge a, Supremo only runs bicubic interpolation on-device and uses a lightweight variant of the IDN [64] SR model to be run on the resource-rich server, while performing patch selection to only transmit key patches. Specifically, Supremo's patch selection mechanism starts by extracting the edges, using the Canny edge detector, from each image, dividing the image into blocks, and sorting the blocks according to edge intensity; the highest edge intensity has the highest priority for offloading, as edges are degraded the most when upscaled using a neural-based model compared to using plain bicubic interpolation. Next, depending on the networking conditions, latency requirements, and their ranking, these patches are sent in parallel to the cloud to be upsampled through the SR model. To further reduce the network footprint required to download the super-resolved patches, Supremo exploits the sparsity of the difference between the super-resolved patches and bicubic-upsampled patches. As these differences are often very sparse, encoding them through the Residual Encoder, which resembles JPEG encoding, results in a heavily compressed signal, thus minimizing bandwidth. For video-centric use-cases, Supremo also deploys a caching mechanism to exploit temporal redundancy by reusing super-resolved frames on matching blocks (Frame Cache), matched using motion estimation [177]. Similar to MobiSR, Supremo handles challenge b by employing a generic model that aims to maximize the average upscaling performance across all processed images.

\subsection{Live Content Streaming Systems}

4.2.1 Streaming for Video Analytics. Pipelines for video analytics [25, 36, 53, 171] perform realtime intelligent tasks over user inputs to enable the development of novel applications such as telepresence [170] and augmented reality apps [106]. Such tasks span from scene labeling and object detection to face recognition. To meet the real-time performance requirements across diverse 

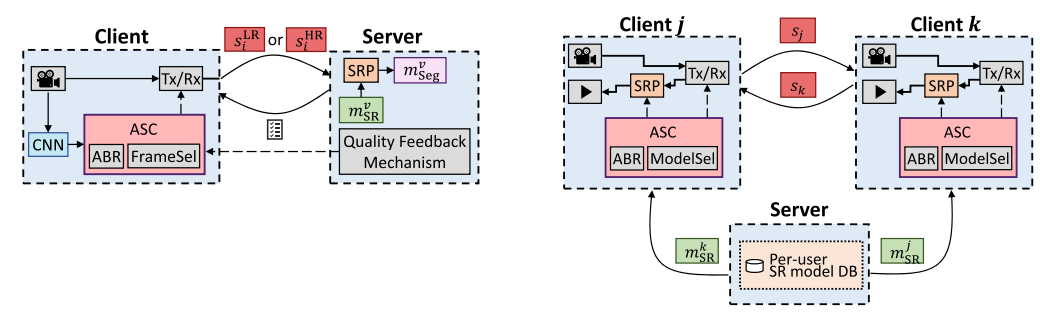

(a)

(b)

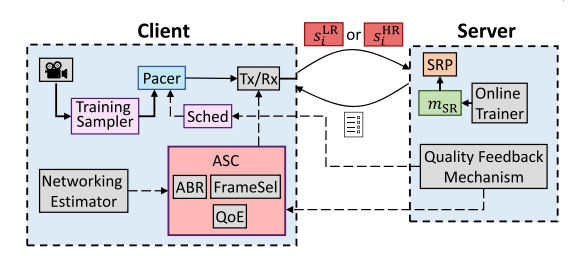

(c)

Fig. 3. Overview of live streaming systems: 3(a)) CloudSeg [152], 3(b)) Dejavu [54], 3(c)) LiveNAS [79]. Cyan blocks indicate novel components introduced by each system.

hardware platforms, such systems often rely on cloud-centric solutions. In this setup, the client device transmits the input frames to a powerful server for analysis and collects back only the result.

Naturally, these video analytics frameworks can benefit from the transmission of lowerresolution or lower-quality images under bandwidth-constrained settings. However, the use of low-quality images is known to reduce accuracy [15,21], as some of these degraded images do not contain the sufficient information for the task at hand. For instance, high-frequency details, such as the texture of an object or the facial identity of distant persons, during downsampling [14]. Therefore, these frameworks can use additional server-side computation to deploy neural enhancement models, recover the quality of the images, and thus minimize the accuracy loss of the target task. To achieve this goal, Wang et al. [152] proposed CloudSeg (Figure 3(a)) that jointly trains an SR model (CARN [3]) together with its target analytics task, i.e., semantic segmentation (ICNet [176]). Specifically, they trained CARN using gradients computed from both the content loss between the $\mathrm{HR}$ and the super-resolved image and the accuracy difference between using both images in ICNet.

During inference, the SRP feeds both the LR image and super-resolved image to the pyramid (also known as multi-scale) segmentation model, avoiding any redundant downsampling process. Towards efficiency (challenge a), CloudSeg employs frame selection at the client side by deploying a small convolutional neural network $(\boldsymbol{C N N})$ that estimates pixel deviation in segmentation maps from low-level features of consecutive frames [96] to skip redundant stale frames (Frame Selector). Finally, CloudSeg overcomes challenge (b by training both the SR and segmentation model on the same dataset and falling back to streaming in high resolution whenever the analytics accuracy falls below a threshold, i.e., the Quality Feedback Mechanism guides the ABR controller.

4.2.2 Video-conferencing. To sustain the interactive communication between callers, videoconfe-rencing requires low response time. In an effort to achieve that, existing services [40] often adopt conservative strategies that relax the bandwidth requirements, but also compromise visual quality.

In this context, $\mathrm{Hu}$ et al. [54] observed that in contrast to generic live streaming, videoconferencing has the property of visual similarity between recurring sessions and designed Dejavu 
(Figure 3(b)) to exploit these unique computational and specialization opportunities; video calls that take place periodically and in the same or similar rooms have inherent redundancy.The developed system starts with the offline training of image enhancement models that are specialized per caller (challenge (b). In this process, the model learns to improve the video quality by increasing its encoding rate without changing the spatial resolution.

Upon deployment, when a video-conference session is established, the associated caller-specific enhancement model is transferred from a client to the other and vice versa $\left(m_{\mathrm{SR}}^{\{k, j\}}\right.$ in Figure 3(b)).During the call, the frames from the caller are re-encoded to a lower quality at the same resolution prior to transmission, reducing the bandwidth usage, and the quality is enhanced on the receiver side through the caller-specific enhancement model.

To address challenge a, Dejavu uses several techniques. Apart from designing an efficient variant of EDSR that is trained only on the luminance channel (Section 3), a powerful GPU is assumed to be available on each calling side. Additionally, Dejavu introduces a patch-scoring CNN that predicts the expected quality gain for each image patch. In this manner, only the top- $k$ patches (Frame Selector), which consist of mainly edges and complex textures, that are expected to yield the highest quality improvement are processed by the quality-enhancing neural network to lower the runtime resource usage.

4.2.3 Live Video Streaming. In contrast to VOD services that focus on stored content, live streaming targets videos produced in real-time. In this case, an additional bottleneck is introduced on the upstream client-to-server channel: A degraded quality from the streaming user would propagate to the end-users that watch the video, leading to additional challenges in sustaining high QoE. Moreover, while stored content in VOD or the recurrence of video-conferencing allows for offline specialization of the enhancement models, the real-time dynamic nature of live streaming requires methods to tailor the models online to the incoming video. Online learning, however, requires extra bandwidth on top of the stream as the parts of the high-quality stream often needs to be transmitted for model fine-tuning.

To tackle these challenges, Kim et al. [79] proposed the LiveNAS system (Figure 3(c)) that focuses on optimizing the upstream transmission from streamer to server.In this system, a generic SR model, pre-trained on previously played similar streams, resides on the server side. Upon deployment, the streamer uploads a series of low-resolution frames that are then enhanced at the server side by the Super-resolution Processor (SRP in Figure 3(c)).

To counteract the performance variability across diverse content (challenge b), Li veNAS introduces an online learning scheme that tailors the model to the particular unseen video. This scheme consists of selectively picking high-quality patches using the Training Sampler and transmitting them from the streamer to the server using the Pacer (Figure 3(c)).Since the patches needed for online training share bandwidth with the video, it is crucial to send only the patches with the highest expected impact. Hence, the Training Sampler detects patches that are hard to compress with high quality, by calculating the PSNR between HR patches and their bilinearly interpolated LR encoding and selecting the lowest-PSNR patches. The Pacer, however, is responsible for allocating the available bandwidth between the low-resolution patches to be upscaled and the high-resolution training patches by adaptively tuning the respective bitrate. This is achieved through a scheduling algorithm (Sched in Figure 3(c)), which aims to optimize the video quality together with the expected online quality gain given the total training patches thus far.

On the server side, the transferred high-resolution patches are used by the Online Trainer to fine-tune the SR model. Further mitigating challenge b) recent patches are weighted more than older patches, better reflecting the current content of the stream. The invocation frequency of the Online Trainer is controlled based on an adaptive mechanism that detects training saturation and 
Table 3. Comparison of Visual Content Delivery Systems

\begin{tabular}{|c|c|c|c|c|}
\hline System & Ref. CNN Model & Computational Optimizations & Model Specialization & CNN Execution \\
\hline MobiSR [92] & RCAN [175] & $\begin{array}{l}\text { (1) Difficulty-aware model selection } \\
\text { (2) Parallel execution }\end{array}$ & Generic model & Client \\
\hline Yeo et al. [165] & VDSR [78] & $\begin{array}{l}\text { (3) Efficient convolutions and upsampling } \\
\text { (1) Powerful desktop clients } \\
\text { (2) Homogeneous clients } \\
\text { (3) Up to } 720 p\end{array}$ & Offline per video category & Client \\
\hline NAS [166] & MDSR [104] & $\begin{array}{l}\text { (1) Powerful desktop clients } \\
\text { (2) Early-exit models } \\
\text { (3) FP16 quantization }\end{array}$ & Offline per video & Client \\
\hline NEMO [164] & MDSR [104] & $\begin{array}{l}\text { (1) Key-frame selection } \\
\text { (2) Early-exit models }\end{array}$ & Offline per video & Client \\
\hline PARSEC [28] & MDSR [104] & $\begin{array}{l}\text { (1) Ultra low-resolution input } \\
\text { (2) Manually-tuned models }\end{array}$ & Offline per video segment & Client \\
\hline Supremo [167] & IDN [64] & $\begin{array}{l}\text { (1) Patch selection } \\
\text { (2) Lightweight model IDN-lite }\end{array}$ & Generic model & Server \\
\hline CloudSeg [152] & CARN [3] & (1) Frame selection & Generic model & Server \\
\hline Dejavu [54] & EDSR [104] & $\begin{array}{l}\text { (1) Patch selection } \\
\text { (2) Luminance-only training } \\
\text { (3) Powerful desktop client }\end{array}$ & Offline per caller & Client \\
\hline LiveNAS [79] & MDSR [104] & $\begin{array}{l}\text { (1) Patch Selection } \\
\text { (2) Parallel execution }\end{array}$ & Online per video & Server \\
\hline SplitSR [108] & RCAN [175] & $\begin{array}{l}\text { (1) Efficient convolutions and model depth } \\
\text { (2) Compiler optimizations for CPU }\end{array}$ & Quality- or latency-optimized & Client \\
\hline
\end{tabular}

scene changes, periodically sending feedback to the client (Quality Feedback Mechanism). Training saturation, in particular, is tracked by measuring the performance gain from the two most recent models, suspending training and sending a signal to the client that sets the patch bitrate to a minimum value if the gain falls under a threshold consecutively. Scene change, however, is identified by comparing the performance between the initial and latest model, resuming online training with recent patches and prompting the client to reset the patch bitrate to its initial value. Thus, the amount of training for each live stream is adapted to maximize performance without excessive resource usage. Finally, to alleviate challenge a, LiveNAS supports scale-out execution by parallelizing the SR computations across multiple GPUs (e.g., three GPUs for 1080p to $4 \mathrm{~K}$ real-time enhancement), if available, on the server.

\subsection{Discussion: Dataset Collection}

A challenging issue of neural enhancement-based CDS systems is the collection of data for the training of the employed DNNs and the end-to-end evaluation of the whole system. Depending on whether the system is image-centric, video-centric, or targets a more specialized application (e.g., $360^{\circ}$ videos, semantic segmentation, or video-conferencing), each CDS employs a different strategy for the data collection stage. Image-centric systems, such as Mobi SR, Supremo, and Spli tSR, employ broadly used super-resolution datasets, including the DIV2K dataset for the training of their DNN models and Set5, Set14, B100, and Urban100 for the evaluation stage. Video-oriented systems, such as NAS, NEMO, and LiveNAS, rely on ad hoc methodologies for the collection of datasets from existing service providers, such as YouTube. Such methodologies typically comprise a selection of the top- $N$ most popular videos from the top- $K$ most popular categories or channels on the service-provider's platform. For instance, NEMO, which focuses on on-demand video delivery, selected three $4 \mathrm{~K}$ videos from the top-10 most popular categories on YouTube, while LiveNAS, which targets live streaming, used the most recent streams from the top streamer in Twitch's five most popular categories and one video for each of the top-four most popular live categories on YouTube. However, PARSEC used the most broadly used $360^{\circ}$ head movement dataset [109] that contains head 
movement from 50 users on $10360^{\circ}$ videos from YouTube. CloudSeg employed the widely used Cityscapes [24], which is a semantic segmentation dataset with urban scenery. Finally, for Dejavu, the authors constructed a proprietary dataset by conducting five mock interviews under the same conditions, i.e., same meeting room on the same day, and recording them using a commodity smartphone on a tripod. Regardless of the dataset used, the respective low-quality counterpart can be synthesized from the original high-resolution image using a uniform degradation operation, such as the widely used bicubic interpolation, to form input-output pairs for supervised training. Although simple, these degradation operations suffice, as the same degradation operations are used during inference. Nonetheless, the choice of dataset would directly affect the visual quality and the upscaling process will be considerably impacted if the data distribution encountered at runtime greatly differs from the training data distribution, resulting in fail-safe mechanisms such as directly sending the HR patches [152]. To counteract this, we provide directions to improve the robustness of these models in Section 5.4.

\subsection{Discussion: Trends in Neural Enhancement-based Content Delivery Systems}

State-of-the-art neural enhancement-based content delivery systems continue to face tougher deployment challenges as compared to earlier systems due to the naturally evolving landscape of various applications and devices. These recent systems have to take into account of a wider range of heterogeneous clients and thus ensure backward compatibility with older devices. On top of that, existing mainstream applications, such as live streaming and video calls, involve dynamic content, exacerbating challenge b. Newly emerging applications such as $360^{\circ}$ videos, in contrast, have unprecedented computational demand to support new immersive experiences at higher resolutions (challenge a). In response to neural enhancement deployment challenges, existing CDS have deployed numerous mitigation techniques, as summarized in Table 3. Although the diversity of target applications, with their unique requirement and challenges, does not allow us to draw conclusive and meaningful results on the performance comparison between the existing neural enhancement-based CDS, the following observations are made:

Frameworks that combine both lightweight and adaptive/scalable neural networks demonstrate higher adaptability. This property can be observed in CDS such as NAS, NEMO, MobiSR, and SplitSR. On the one hand, NAS and NEMO employ: (1) a lightweight model design through a compact variant of MDSR [104] with 16-bit quantization, together with (2) a per-video catalog of models with diverse complexity-performance tradeoffs that enables the static model selection based on the client device nominal capabilities, and (3) a scalable design of individual models that enables the $d y$ namic adaptation of model complexity to the runtime device load. Adopting an alternative approach, MobiSR combines: (1) a lightweight compressed version of RCAN [175], together with (2) a static model selection based on the target device capabilities and model performance, and (3) a runtime model selection scheduler that pins a distinct model to each compute engine of the target platform and dispatches each patch to the suitable model-engine pair. Similarly, SplitSR employs a customized version of RCAN, optimized for either quality or latency. Such approaches provide greater flexibility at both design and runtime. In this manner, CDS have more room to adapt to static (e.g., device capabilities) and dynamic constraints (e.g., device load, networking conditions), meet the latency and visual quality requirements, and maximize QoE.

Applying informed frame/patch-selective enhancement through a quality-aware mechanism tends to radically improve QoE with minimal visual quality degradation. NEMO and Supremo's caches that store previously super-resolved frames enables the reuse of already computed results, thus skipping the costly processing overhead of neural enhancement. With the goal to sustain high visual quality in the challenging task of $360^{\circ}$ video delivery, PARSEC selectively chooses which frame 
patches to enhance and which to directly download in high resolution. A similar strategy is adopted by CloudSeg with the aim to maximize the accuracy of the target semantic segmentation task. In another direction, LiveNAS employs frame selection to determine which high-resolution frames should be transmitted to the server side for further neural model fine-tuning on the current live stream. Across these CDS, the frame/patch selection criteria aim to either select content based on their upscaling or encoding difficulty or the degree of temporal redundancy. For instance, patches with complex textures and edges are often prioritized to be enhanced to maximize performance, or frames with high correlations with subsequent frames are cached after enhancement to be reused.

Specializing models to specific content tends to reduce the complexity of neural model design and rely more on system-level solutions. General mitigations to challenge b involve fine-tuning from a pre-trained generic model to the targeted content per scale for each client's computational regime. With the exemption of MobiSR, Supremo, and CloudSeg, all existing CDS adopt a variant of this model specialization approach. These systems require $C * S * \epsilon$ trained models where $C$ is the number of unique content (i.e., entire video, video cluster, or video segment), $S$ is the number of available scales for each content, and $\epsilon$ is the number of supported computational regimes across its clients (e.g., low-end, mid-tier, and high-end platforms). This computational load is a one-time cost for offline applications, but imposes additional challenges for their online counterparts, such as LiveNAS, as discussed in Section 4.2. This approach relieves CDS designers from designing sophisticated neural enhancement models by deriving multiple specialized and scale-specific variants from a single reference model, with the CDS deploying the suitable one as requested by the client. Nevertheless, it leaves space for further model-level exploration, as discussed in Section 5.2.

\section{FUTURE DIRECTIONS}

In this section, we propose various approaches that are drawn from the latest computer vision research and provide insights on how neural enhancement can further benefit content delivery systems. Specifically, we highlight promising directions to further address the challenges of neural enhancement.

\subsection{Improving Visual Quality}

One of the main open challenges in neural enhancement algorithms is the design of a metric that will correspond well with human raters. As mentioned in Section 2.1, distortion-based metrics, such as PSNR [44] or SSIM [155], which aim to minimize the per-pixel error between two images, have been extensively shown to improve image fidelity at the cost of perceptual quality, leading to blurry and unnatural outcomes [88]. However, optimizing only for a perceptual-based metric such as Naturalness Image Quality Evaluator (NIQE) [117] and Learned Perceptual Image Patch Similarity (LPIPS) [174] will lead to more natural-looking images at the expense of fidelity and therefore the occasional occurrence of image artefacts. Mathematically, there is a tradeoff between fidelity and perceptual quality [13].

As all the existing neural enhancement frameworks (Section 4) train their models using a distortion-based metric, the outputs of these models are accurate, but may look unnatural. Although this will benefit video analytics frameworks, such as CloudSeg, having blurry outputs will undermine the goal of other content streaming systems, such as Dejavu and LiveNAS. To close this gap, these systems can benefit further by utilizing recent methods proposed in computer vision to train and optimize their neural enhancement models. Some of these works focus on striking an optimal point between image fidelity and perceptual quality by optimizing for both distortion-based and perceptual-based metrics [91, 151]. Specifically, these works often optimize their models jointly on L1/Mean-Squared-Error (MSE) losses for image fidelity and a variety 
of losses, including perceptual loss [73], adversarial loss [45], and contextual loss [116], for perceptual quality. Additionally, other methods such as interpolating between a distortion-based and perceptual-based output image [31] or model [150, 151], or introducing additional priors [111, 133], can also be used to alleviate undesirable artefacts caused by optimizing only for perception-based metrics. For instance, utilizing rich texture priors found in pre-trained generative models has been shown to result in images that are high in both fidelity and perceptual quality [16]. Although the aforementioned works have found success in achieving high visual quality content, most propose techniques and models that are too computationally demanding to be deployed in existing CDS. Hence, there are potential gaps in the literature to realize the benefits of utilizing these approaches.

For video-based solutions, the majority of existing vision works utilize temporal information by aligning and fusing spatial information from multiple frames to further boost image fidelity $[17,75,102,127]$. Specifically, these CNN- or RNN-based solutions align adjacent frames by warping each supporting frame to a reference frame using the respective optical flow. These optical flows are usually estimated using either traditional motion estimation algorithms [8] or CNNbased approaches, such as spatial transformer networks [69] and task-specific motion estimation networks $[66,125,139]$. Instead of utilizing an explicit component for motion estimation, another line of work $[72,143,148]$ has proposed performing alignment implicitly through deformable convolutions $[27,178]$ or dynamically generated filters.

As a result of effectively utilizing multiple frames to upscale each frame, these multi-frame approaches are able to achieve better restoration performance as compared to their singleimage counterparts. Besides improving the visual quality spatially, temporal interpolation methods $[158,180]$ can enable a system to increase the achieved frame rate, and hence the QoE, by estimating intermediate frames rather than transferring them and applying super-resolution. In this manner, the bandwidth requirements are reduced by cutting both the content's spatial and temporal resolution. Nonetheless, video-based enhancement solutions are more computationally demanding than image-based solutions due to additional processing in the temporal domain. Therefore, these techniques can be utilized more effectively in CDS such as NAS and CloudSeg, which perform their computations on powerful desktop clients and on server-grade processors, respectively.

With regards to QoE, the actual bitrate or an estimated bitrate, given the performance of the model, is used to quantify the content's visual quality in relations to the user's experience (Section 2.1). As the amount of computational resources continue to grow in commodity hardware, the content's spatial quality will be largely dependent on the performance of the model. Therefore, there is a need for better designs of the QoE metric to reflect these changes for neural enhancement-based CDS.

\subsection{Utilizing Efficiency-optimized Models}

Most neural enhancement frameworks generally adopt popular full-blown SR models (Table 3) and revise them to speed up training and inference or fit into the limiting constraints for clientside computation. However, these revisions are usually suboptimal or, in some cases, detrimental. For instance, PARSEC uses of batch normalization [67] to speed up training, reducing image fidelity [104] and introducing image artefacts [151]. Therefore, instead of naively scaling down and modifying full-blown SR models, these systems can leverage existing off-the-shelf efficient models that are specifically optimized for both performance and efficiency to deliver higher-quality enhancement at a lower computational cost (challenge a). These models include manually designed variants such as IDN [64]-already used by Supremo, automatically designed variants such as ESRN [134] and TPSR [91] through neural architecture search [179], or even quantized [93] and binarized SR models $[113,160]$ if the hardware supports their efficient execution. 


\subsection{Faster Model Specialization through Meta-learning}

To mitigate challenge b), some systems specialize a model for each specific image/video through overfitting. This approach results in a computationally expensive process (challenge a), as each model requires thousands of gradient updates to be adequately specialized. In response, later systems introduce a two-stage approach. First, a generic neural enhancement model is pre-trained offline, and then fine-tuning is applied either offline or online. For instance, NAS first trains a generic model on an external dataset before using its weights to fine-tune a separate model for each video, amortizing in this manner the one-time offline training cost.

To further speed up and improve the performance during the fine-tuning step, these works can adopt a meta-learning approach [51] to find a more optimal set of initialization parameters for fine-tuning. In other words, pre-training a neural enhancement model via meta-learning on an external dataset will require fewer gradient updates during the fine-tuning stage, therefore requiring fewer computational resources and leading to higher performance compared to bruteforce fine-tuning $[121,132]$.

This fast adaptation is achieved by training the model so it is easy to fine-tune. Specifically, besides having a regular training loop that optimizes the model on an external dataset, there is an additional inner loop that computes the adapted parameters given a different set of paired images. The main set of parameters is then iteratively updated based on the derivative of the loss with respect to the adapted parameters, resulting in a set of parameters that can quickly adapt to a test image during inference. Therefore, the quick adaption of the meta-trained parameters can further save the computations required to fine-tune in both offline (e.g., on-demand streaming) and online cases (e.g., live streaming).

\subsection{Improving Robustness through Conditional Enhancement}

Originally, the usage of SR was to improve the quality of a low-quality image under the assumption that the high-quality version was not available. As such, one of the key benefits of deploying an SR model as a neural enhancement unit is its ability to work without the high-resolution ground-truth. However, in the context of many content delivery settings, the ground-truth is available and the service provider scales it down to a lower quality to minimize the transmission cost. Therefore, instead of discarding the high-frequency information during downsampling, this information can be encoded in a latent variable and transmitted, along with the low-quality frame, to the receiver in CDS. The upsampling process can then be conditioned on that variable, therefore counteracting performance variability (challenge (b) by allowing the same model to better handle differing content instead of having to overfit one model for each content. To this end, several computer vision works leverage the downscaling process during the upscaling process using neural image rescaling techniques to further boost image reconstruction. For instance, a downscaling CNN can be trained jointly with an existing SR model, as shown in Reference [77], and techniques such as encoder-decoder frameworks and invertible neural networks can also be utilized, as shown in References [95] and [159], respectively.

Despite its benefits, neural-based image rescaling incurs an additional cost of executing a downscaling neural network as compared to that of interpolation methods, utilizing additional computational resources for a more robust improvement in visual quality. Therefore, image rescaling techniques may be more suitable for on-demand video systems, such as NAS and PARSEC, in which the downscaling cost is an offline one-time cost across videos. Additionally, although these approaches have not been shown to fully mitigate challenge b. they can significantly reduce the dependency of having to frequently transmit model segments for each content. Ultimately, as models get more robust to varying inputs, they can be deployed once across varying content and even across various applications. 


\subsection{Dynamic Deep Neural Networks}

A growing body of work in the computer vision literature is investigating the design and deployment of dynamic deep neural networks. Such models employ conditional execution mechanisms to provide a runtime tunable accuracy-complexity tradeoff by scaling up and down their computational complexity. Such mechanisms would allow CDS to dynamically adapt the execution of neural enhancement models based on the client device capabilities and compute load and provide another configurable dimension for CDS to ensure high QoE. Nevertheless, the majority of existing work focuses on classification models [38, 42, 48, 53, 55, 74, 76, 82, 83, 86, 87, 128, 142, 149, 154], rather than super-resolution and image enhancement.

Although NAS [166] and MobiSR [92] explored this direction by introducing the scalable NASMDSR model and a difficulty-aware model selection scheme, respectively, the potential of other dynamic methods has remained unexplored. So far, the computer vision community has proposed a broader range of dynamic neural network techniques, spanning from dynamic pruning schemes that skip either layers [149] or channels [38, 42, 55, 153] in an input-dependent manner, model cascades [48, 53, 74, 82, 83, 128], and early-exit models (either hand-crafted [57, 173], hardwareaware [87], distributed [86, 154], or generic [76, 142]). By adapting and modifying such techniques, system designers can develop dynamic neural enhancement models [161] that can be highly optimized specifically for the deployment use-cases of content delivery systems.

\subsection{Hardware Acceleration through Neural Processing Units}

Another promising approach to alleviate the high computational demands of neural enhancement models is targeting the problem from a hardware perspective. At the moment, device vendors have integrated specialized hardware units-often named neural processing units-that are optimized for fast deep neural network processing in both smartphones [65] and embedded platforms [6]. To surpass the limitations of CPUs and GPUs and further boost the processing speed of neural models, several works have proposed custom hardware accelerators [135, 145].

In this context, by considering the unique needs of content delivery systems and their use-cases, highly customized hardware accelerators can be designed. This approach would tailor the underlying processing platform to the CDS' performance requirements, overcoming the performance and energy-efficiency bottlenecks of conventional CPUs and GPUs, and thus ensure high QoE at a lower overall cost. Such custom hardware solutions can be obtained either through hand-crafted designs [50] and super-resolution neural processing units [89], automated accelerator generation tools [147], or sophisticated model-hardware co-design frameworks [1, 19, 80] for the joint development of the neural enhancement model and its efficient hardware accelerator.

\section{CONCLUSION}

This article presents a survey of a new class of visual content delivery systems that employ neural enhancement techniques to boost their performance. Through a detailed analysis of how they tackle the deployment challenges of deep neural enhancement models, we highlight their design choices in terms of system architecture, novel components, and neural model design, and indicate their strengths and weaknesses. Despite the rapid progress of recent systems, the demand for visual content traffic will grow over the coming years due to both existing and emerging technologies, such as augmented and virtual reality $[70,106]$ and telepresence [170]. To this end, based on recent developments from both the computer vision and systems communities, we identify key optimization opportunities and propose promising research directions to address emerging challenges of the field, enhancing their performance and enabling a wider large-scale deployment of high-QoE content delivery services. 


\section{REFERENCES}

[1] Mohamed S. Abdelfattah, Łukasz Dudziak, Thomas Chau, Royson Lee, Hyeji Kim, and Nicholas D. Lane. 2020. Best of both worlds: AutoML codesign of a CNN and its hardware accelerator. In Proceedings of the Design Automation Conference (DAC'20).

[2] Adnan Ahmed, Zubair Shafiq, and Amir Khakpour. 2016. QoE analysis of a large-scale live video streaming event. In Proceedings of the ACM SIGMETRICS International Conference on Measurement and Modeling of Computer Science (SIGMETRICS'16). 395-396.

[3] Namhyuk Ahn, Byungkon Kang, and Kyung-Ah Sohn. 2018. Fast, accurate, and lightweight super-resolution with cascading residual network. In Proceedings of the European Conference on Computer Vision (ECCV'18).

[4] Zahaib Akhtar, Yun Seong Nam, Ramesh Govindan, Sanjay Rao, Jessica Chen, Ethan Katz-Bassett, Bruno Ribeiro, Jibin Zhan, and Hui Zhang. 2018. Oboe: Auto-tuning video ABR algorithms to network conditions. In Proceedings of the Conference of the ACM Special Interest Group on Data Communication (SIGCOMM'18). 44-58.

[5] Mario Almeida, Muhammad Bilal, Alessandro Finamore, Ilias Leontiadis, Yan Grunenberger, Matteo Varvello, and Jeremy Blackburn. 2018. CHIMP: Crowdsourcing human inputs for mobile phones. In Proceedings of the World Wide Web Conference (WWW'18). International World Wide Web Conferences Steering Committee, 45-54.

[6] Mario Almeida, Stefanos Laskaridis, Ilias Leontiadis, Stylianos I. Venieris, and Nicholas D. Lane. 2019. EmBench: Quantifying performance variations of deep neural networks across modern commodity devices. In Proceedings of the 3rd International Workshop on Deep Learning for Mobile Systems and Applications (EMDL'19). 6.

[7] Ghufran Baig, Jian He, Mubashir Adnan Qureshi, Lili Qiu, Guohai Chen, Peng Chen, and Yinliang Hu. 2019. Jigsaw: Robust live $4 \mathrm{~K}$ video streaming. In Proceedings of the 25th International Conference on Mobile Computing and Networking (MobiCom'19).

[8] S. Baker, D. Scharstein, J. Lewis, S. Roth, Michael J. Black, and R. Szeliski. 2011. A database and evaluation methodology for optical flow. Int. fournal of Computer Vision 92, 1 (2011), 1-31.

[9] Sefi Bell-Kligler, Assaf Shocher, and Michal Irani. 2019. Blind super-resolution kernel estimation using an internalGAN. In Proceedings of the International Conference on Advances in Neural Information Processing Systems.

[10] Ibrahim Ben Mustafa, Tamer Nadeem, and Emir Halepovic. 2018. FlexStream: Towards flexible adaptive video streaming on end devices using extreme SDN. In Proceedings of the 26th ACM International Conference on Multimedia (MM’18). 555-563.

[11] A. Bentaleb, B. Taani, A. C. Begen, C. Timmerer, and R. Zimmermann. 2019. A survey on bitrate adaptation schemes for streaming media over HTTP. IEEE Commun. Surv. Tutor. 21, 1 (2019), 562-585.

[12] Marco Bevilacqua, Aline Roumy, Christine Guillemot, and Marie line Alberi Morel. 2012. Low-complexity singleimage super-resolution based on nonnegative neighbor embedding. In Proceedings of the British Machine Vision Conference.

[13] Yochai Blau and Tomer Michaeli. 2018. The perception-distortion tradeoff. In Proceedings of the IEEE/CVF Conference on Computer Vision and Pattern Recognition (CVPR'18).

[14] Dingding Cai, Ke Chen, Y. Qian, and J. Kämäräinen. 2019. Convolutional low-resolution fine-grained classification. Pattern Recog. Lett. 119 (2019), 166-171.

[15] Christopher Canel, Thomas Kim, Giulio Zhou, Conglong Li, Hyeontaek Lim, David G. Andersen, Michael Kaminsky, and Subramanya R. Dulloor. 2019. Scaling video analytics on constrained edge nodes. In Proceedings of the Conference on Machine Learning and Systems (MLSys'19).

[16] Kelvin C. K. Chan, Xintao Wang, Xiangyu Xu, Jinwei Gu, and Chen Change Loy. 2021. GLEAN: Generative latent bank for large-factor image super-resolution. In Proceedings of the IEEE/CVF Conference on Computer Vision and Pattern Recognition (CVPR'21).

[17] Kelvin C. K. Chan, Xintao Wang, Ke Yu, Chao Dong, and Chen Change Loy. 2021. BasicVSR: The search for essential components in video super-resolution and beyond. In Proceedings of the IEEE Conference on Computer Vision and Pattern Recognition (CVPR'21).

[18] Chun-Ming Chang, Cheng-Hsin Hsu, Chih-Fan Hsu, and Kuan-Ta Chen. 2016. Performance measurements of virtual reality systems: Quantifying the timing and positioning accuracy. In Proceedings of the 24th ACM International Conference on Multimedia (MM'16). 655-659.

[19] T. Chau, L. Dudziak, M. Abdelfattah, Royson Lee, H. Kim, and N. Lane. 2020. BRP-NAS: Prediction-based NAS using GCNs. In Proceedings of the International Conference on Advances in Neural Information Processing Systems (NeurIPS'20).

[20] Tianqi Chen, Thierry Moreau, Ziheng Jiang, Lianmin Zheng, Eddie Yan, Haichen Shen, Meghan Cowan, Leyuan Wang, Yuwei Hu, Luis Ceze, Carlos Guestrin, and Arvind Krishnamurthy. 2018. TVM: An automated end-to-end optimizing compiler for deep learning. In Proceedings of the 13th USENIX Symposium on Operating Systems Design and Implementation (OSDI'18). 578-594. 
[21] T. Chen, L. Ravindranath, Shuo Deng, P. Bahl, and H. Balakrishnan. 2015. Glimpse: Continuous, real-time object recognition on mobile devices. In Proceedings of the SenSys Conference.

[22] Cisco. 2020. Cisco Annual Internet Report (2018-2023) White Paper. Technical Report. Cisco Systems, Inc. Retrieved from https:/www.cisco.com/c/en/us/solutions/collateral/executive-perspectives/annual-internet-report/ white-paper-c11-741490.html.

[23] Cisco. 2020. Cisco Visual Networking Index (VNI) Complete Forecast Update, 2017-2022. Technical Report. Cisco Systems, Inc. Retrieved from https://www.cisco.com/c/dam/m/en_us/network-intelligence/service-provider/digitaltransformation/knowledge-network-webinars/pdfs/1213-business-services-ckn.pdf.

[24] Marius Cordts, Mohamed Omran, Sebastian Ramos, Timo Rehfeld, Markus Enzweiler, Rodrigo Benenson, Uwe Franke, Stefan Roth, and Bernt Schiele. 2016. The cityscapes dataset for semantic urban scene understanding. In Proceedings of the IEEE Conference on Computer Vision and Pattern Recognition (CVPR'16).

[25] Daniel Crankshaw, Gur-Eyal Sela, Xiangxi Mo, Corey Zumar, Ion Stoica, Joseph Gonzalez, and Alexey Tumanov. 2020. InferLine: Latency-aware provisioning and scaling for prediction serving pipelines. In Proceedings of the 11th ACM Symposium on Cloud Computing (SoCC'20). 477-491.

[26] Simon Da Silva, Sonia Ben Mokhtar, Stefan Contiu, Daniel Négru, Laurent Réveillère, and Etienne Rivière. 2019. PrivaTube: Privacy-preserving edge-assisted video streaming. In Proceedings of the 20th International Middleware Conference (Middleware'19). 189-201.

[27] Jifeng Dai, Haozhi Qi, Y. Xiong, Y. Li, Guodong Zhang, H. Hu, and Y. Wei. 2017. Deformable convolutional networks.In Proceedings of the IEEE International Conference on Computer Vision (ICCV'17).

[28] Mallesham Dasari, A. Bhattacharya, Santiago Vargas, Pranjal Sahu, A. Balasubramanian, and S. Das. 2020. Streaming 360-degree videos using super-resolution. In Proceedings of the IEEE Conference on Computer Communications (INFOCOM'20).

[29] L. De Cicco, V. Caldaralo, V. Palmisano, and S. Mascolo. 2013. ELASTIC: A client-side controller for dynamic adaptive streaming over HTTP (DASH). In Proceedings of the 20th International Packet Video Workshop. 1-8.

[30] Jonathan Deber, Ricardo Jota, Clifton Forlines, and Daniel Wigdor. 2015. How much faster is fast enough? User perception of latency \& latency improvements in direct and indirect touch. In Proceedings of the 33rd ACM Conference on Human Factors in Computing Systems (CHI'15). 1827-1836.

[31] Xin Deng. 2018. Enhancing image quality via style transfer for single image super-resolution. IEEE Sig. Process. Lett. 25, 4 (2018), 571-575.

[32] Giorgos Dimopoulos, Ilias Leontiadis, Pere Barlet-Ros, and Konstantina Papagiannaki. 2016. Measuring video QoE from encrypted traffic. In Proceedings of the Internet Measurement Conference (IMC'16). 513-526.

[33] Pradeep Dogga, Sandip Chakraborty, Subrata Mitra, and Ravi Netravali. 2019. Edge-based transcoding for adaptive live video streaming. In Proceedings of the 2nd USENIX Workshop on Hot Topics in Edge Computing (HotEdge'19). USENIX Association.

[34] Chao Dong, Chen Change Loy, Kaiming He, and Xiaoou Tang. 2016. Image super-resolution using deep convolutional networks. IEEE Trans. Pattern Anal. Mach. Intell. 38, 2 (2016), 295-307.

[35] Chao Dong, Chen Change Loy, and Xiaoou Tang. 2016. Accelerating the super-resolution convolutional neural network. In Proceedings of the European Computer on Computer Vision (ECCV'16).

[36] Kuntai Du, Ahsan Pervaiz, Xin Yuan, Aakanksha Chowdhery, Qizheng Zhang, Henry Hoffmann, and J. Jiang. 2020. Server-driven video streaming for deep learning inference. In Proceedings of the SIGCOMM Conference.

[37] Ching-Ling Fan, J. Lee, Wen-Chih Lo, C. Huang, Kuan-Ta Chen, and C. Hsu. 2017. Fixation prediction for 360 video streaming in head-mounted virtual reality. In Network and Operating System Support for Digital Audio and Video. Association for Computing Machinery, 67-72.

[38] Biyi Fang, Xiao Zeng, and Mi Zhang. 2018. NestDNN: Resource-aware multi-tenant on-device deep learning for continuous mobile vision. In Proceedings of the 24th International Conference on Mobile Computing and Networking (MobiCom'18). 115-127.

[39] M. Fiedler, T. Hossfeld, and P. Tran-Gia. 2010. A generic quantitative relationship between quality of experience and quality of service. IEEE Netw. 24, 2 (2010), 36-41.

[40] Sadjad Fouladi, John Emmons, Emre Orbay, C. Wu, Riad S. Wahby, and Keith Winstein. 2018. Salsify: Low-latency network video through tighter integration between a video codec and a transport protocol. In Proceedings of the 15th USENIX Symposium on Networked Systems Design and Implementation (NSDI'18).

[41] M. Gadaleta, F. Chiariotti, M. Rossi, and A. Zanella. 2017. D-DASH: A deep q-learning framework for DASH video streaming. IEEE Trans. Cognit. Commun. Netw. 3, 4 (2017), 703-718.

[42] Xitong Gao, Yiren Zhao, Łukasz Dudziak, Robert Mullins, and Cheng Zhong Xu. 2019. Dynamic channel pruning: Feature boosting and suppression. In Proceedings of the International Conference on Learning Representations.

[43] C. Ge, N. Wang, G. Foster, and M. Wilson. 2017. Toward QoE-assured 4K video-on-demand delivery through mobile edge virtualization with adaptive prefetching. IEEE Trans. Multimedia 19, 10 (2017), 2222-2237. 
[44] Rafael C. Gonzalez and Richard E. Woods. 2008. Digital Image Processing. Prentice Hall, Upper Saddle River, N.J.

[45] Ian Goodfellow et al. 2014. Generative adversarial nets. In Proceedings of the International Conference on Advances in Neural Information Processing Systems (NeurIPS'14).

[46] Jinjin Gu, Hannan Lu, W. Zuo, and C. Dong. 2019. Blind super-resolution with iterative kernel correction. In Proceedings of the IEEE/CVF Conference on Computer Vision and Pattern Recognition (CVPR'19).

[47] T. Guarnieri, I. Drago, A. B. Vieira, I. Cunha, and J. Almeida. 2017. Characterizing QoE in large-scale live streaming. In Proceedings of the IEEE Global Communications Conference (GLOBECOM'17). 1-7.

[48] Seungyeop Han, Haichen Shen, Matthai Philipose, Sharad Agarwal, Alec Wolman, and Arvind Krishnamurthy. 2016. MCDNN: An approximation-based execution framework for deep stream processing under resource constraints. In Proceedings of the 14th International Conference on Mobile Systems, Applications, and Services (MobiSys'16).

[49] Kaiming He, X. Zhang, Shaoqing Ren, and Jian Sun. 2016. Deep residual learning for image recognition.In Proceedings of the IEEE Conference on Computer Vision and Pattern Recognition (CVPR'16).

[50] Z. He, H. Huang, M. Jiang, Y. Bai, and G. Luo. 2018. FPGA-based real-time super-resolution system for ultra high definition videos. In Proceedings of the IEEE 26th International Symposium on Field-programmable Custom Computing Machines (FCCM'18). 181-188.

[51] Timothy Hospedales, Antreas Antoniou, Paul Micaelli, and Amos Storkey. 2020. Meta-Learning in Neural Networks: A Survey. arXiv:cs.LG/2004.05439.

[52] A. Howard, Mark Sandler, G. Chu, Liang-Chieh Chen, B. Chen, M. Tan, W. Wang, Y. Zhu, R. Pang, V. Vasudevan, Quoc V. Le, and H. Adam. 2019. Searching for mobileNetV3.In Proceedings of the International Conference on Computer Vision (ICCV'19).

[53] Kevin Hsieh, Ganesh Ananthanarayanan, Peter Bodik, Shivaram Venkataraman, Paramvir Bahl, Matthai Philipose, Phillip B. Gibbons, and Onur Mutlu. 2018. Focus: Querying large video datasets with low latency and low cost. In Proceedings of the 12th USENIX Conference on Operating Systems Design and Implementation (OSDI'18). 269-286.

[54] Pan Hu, Rakesh Misra, and Sachin Katti. 2019. Dejavu: Enhancing videoconferencing with prior knowledge. In Proceedings of the HotMobile Conference.

[55] Weizhe Hua, Yuan Zhou, Christopher M. De Sa, Zhiru Zhang, and G. Edward Suh. 2019. Channel gating neural networks. In Proceedings of the International Conference on Advances in Neural Information Processing Systems (NeurIPS'19). 1886-1896.

[56] Cheng Huang, Jin Li, and Keith W. Ross. 2007. Can internet video-on-demand be profitable? In Proceedings of the Conference on Applications, Technologies, Architectures, and Protocols for Computer Communications (SIGCOMM'07). 133-144.

[57] Gao Huang, Danlu Chen, Tianhong Li, Felix Wu, Laurens van der Maaten, and Kilian Weinberger. 2018. Multi-scale dense networks for resource efficient image classification. In Proceedings of the International Conference on Learning Representations (ICLR'18).

[58] Gao Huang, Zhuang Liu, and K. Weinberger. 2017. Densely connected convolutional networks.In Proceedings of the IEEE Conference on Computer Vision and Pattern Recognition (CVPR'17).

[59] J. Huang, A. Singh, and N. Ahuja. 2015. Single image super-resolution from transformed self-exemplars. In Proceedings of the IEEE Conference on Computer Vision and Pattern Recognition (CVPR'15).

[60] Tianchi Huang, Rui-Xiao Zhang, Chao Zhou, and Lifeng Sun. 2018. QARC: Video quality aware rate control for realtime video streaming based on deep reinforcement learning. In Proceedings of the 26th ACM International Conference on Multimedia (MM'18). 1208-1216.

[61] T. Huang, C. Zhou, X. Yao, R. X. Zhang, C. Wu, B. Yu, and L. Sun. 2020. Quality-aware neural adaptive video streaming with lifelong imitation learning. IEEE 7. Select. Areas Commun. 38, 10 (2020), 2324-2342.

[62] Te-Yuan Huang, Chaitanya Ekanadham, Andrew J. Berglund, and Zhi Li. 2019. Hindsight: Evaluate video bitrate adaptation at scale. In Proceedings of the 10th ACM Multimedia Systems Conference (MMSys'19). Association for Computing Machinery, New York, NY, 86-97. DOI : https://doi.org/10.1145/3304109.3306219.

[63] Te-Yuan Huang, Ramesh Johari, Nick McKeown, Matthew Trunnell, and Mark Watson. 2014. A buffer-based approach to rate adaptation: Evidence from a large video streaming service. In Proceedings of the SIGCOMM Conference.

[64] Zheng Hui, Xiumei Wang, and Xinbo Gao. 2018. Fast and accurate single image super-resolution via information distillation network. In Proceedings of the IEEE/CVF Conference on Computer Vision and Pattern Recognition (CVPR'18).

[65] Andrey Ignatov, Radu Timofte, Andrei Kulik, Seungsoo Yang, Ke Wang, Felix Baum, Max Wu, Lirong Xu, and Luc Van Gool. 2019. AI benchmark: All about deep learning on smartphones in 2019. In Proceedings of the International Conference on Computer Vision (ICCV'19) Workshops.

[66] Eddy Ilg, N. Mayer, Tonmoy Saikia, Margret Keuper, A. Dosovitskiy, and T. Brox. 2017. FlowNet 2.0: Evolution of optical flow estimation with deep networks. In Proceedings of the IEEE Conference on Computer Vision and Pattern Recognition (CVPR'17). 
[67] S. Ioffe and Christian Szegedy. 2015. Batch normalization: Accelerating deep network training by reducing internal covariate shift. In Proceedings of the International Conference on Machine Learning (ICML'15).

[68] ITU. 2008. ITU-T Recommendation P.910. Subjective video quality assessment methods for multimedia applications. Retrieved from https://www.itu.int/rec/T-REC-P.910-200804-I.

[69] Max Jaderberg, K. Simonyan, Andrew Zisserman, and K. Kavukcuoglu. 2015. Spatial transformer networks. In Proceedings of the International Conference on Advances in Neural Information Processing Systems.

[70] Puneet Jain, Justin Manweiler, and Romit Roy Choudhury. 2015. OverLay: Practical mobile augmented reality. In Proceedings of the 13th International Conference on Mobile Systems, Applications, and Services (MobiSys'15).

[71] J. Jiang, V. Sekar, and H. Zhang. 2014. Improving fairness, efficiency, and stability in HTTP-based adaptive video streaming with festive. IEEE/ACM Trans. Netw. 22, 1 (2014), 326-340.

[72] Younghyun Jo, S. Oh, Jaeyeon Kang, and S. Kim. 2018. Deep video super-resolution network using dynamic upsampling filters without explicit motion compensation.In Proceedings of the IEEE/CVF Conference on Computer Vision and Pattern Recognition.

[73] Justin Johnson, Alexandre Alahi, and Li Fei-Fei. 2016. Perceptual losses for real-time style transfer and superresolution. In Proceedings of the European Conference on Computer Vision (ECCV'16).

[74] Daniel Kang, John Emmons, Firas Abuzaid, Peter Bailis, and Matei Zaharia. 2017. NoScope: Optimizing neural network queries over video at scale. Proc. VLDB Endow. 10, 11 (2017), 1586-1597.

[75] Armin Kappeler, Seunghwan Yoo, Qiqin Dai, and A. Katsaggelos. 2016. Video super-resolution with convolutional neural networks. IEEE Trans. Computat. Imag. 2, 2 (2016), 109-122.

[76] Yigitcan Kaya, Sanghyun Hong, and Tudor Dumitras. 2019. Shallow-deep networks: Understanding and mitigating network overthinking. In Proceedings of the International Conference on Machine Learning (ICML'19), Vol. 97. PMLR, 3301-3310.

[77] Heewon Kim, Myungsub Choi, Bee Lim, and Kyoung Mu Lee. 2018. Task-aware image downscaling. In Proceedings of the European Conference on Computer Vision (ECCV'18).

[78] Jiwon Kim, Jung Kwon Lee, and Kyoung Mu Lee. 2016. Accurate image super-resolution using very deep convolutional networks. In Proceedings of the IEEE/CVF Conference on Computer Vision and Pattern Recognition (CVPR'16).

[79] Jae-Hong Kim, Youngmok Jung, H. Yeo, Juncheol Ye, and D. Han. 2020. Neural-enhanced live streaming: Improving live video ingest via online learning. In Proceedings of the SIGCOMM Conference.

[80] Y. Kim, J. Choi, and M. Kim. 2018. A real-time convolutional neural network for super-resolution on FPGA with applications to 4K UHD 60 fps video services. IEEE Trans. Circ. Syst. Vid. Technol. 29, 8 (2018), 2521-2534.

[81] G. T. Kolyvas, S. E. Polykalas, and I. S. Venieris. 1997. Performance evaluation of intelligent signaling servers for broadband multimedia networks. In Proceedings 2nd IEEE Symposium on Computer and Communications (ICC'97). 96-103.

[82] A. Kouris, S. I. Venieris, and C. Bouganis. 2020. A throughput-latency co-optimised cascade of convolutional neural network classifiers. In Proceedings of the Design, Automation Test in Europe Conference Exhibition (DATE'20). 16561661.

[83] A. Kouris, S. I. Venieris, and C. S. Bouganis. 2018. CascadeCNN: Pushing the performance limits of quantisation in convolutional neural networks. In Proceedings of the 28th International Conference on Field Programmable Logic and Applications (FPL'18).

[84] Alex Krizhevsky, Ilya Sutskever, and Geoffrey E. Hinton. 2012. ImageNet classification with deep convolutional neural networks. In Proceedings of the International Conference on Advances in Neural Processing Systems (NeurIPS'12).

[85] N. D. Lane, S. Bhattacharya, A. Mathur, P. Georgiev, C. Forlivesi, and F. Kawsar. 2017. Squeezing deep learning into mobile and embedded devices. IEEE Pervas. Comput. 16, 3 (2017), 82-88.

[86] Stefanos Laskaridis, Stylianos I. Venieris, Mario Almeida, Ilias Leontiadis, and Nicholas D. Lane. 2020. SPINN: Synergistic progressive inference of neural networks over device and cloud. In Proceedings of the 26th International Conference on Mobile Computing and Networking (MobiCom'20).

[87] Stefanos Laskaridis, Stylianos I. Venieris, Hyeji Kim, and Nicholas D. Lane. 2020. HAPI: Hardware-aware progressive inference. In Proceedings of the IEEE/ACM International Conference on Computer-Aided Design (ICCAD'20).

[88] C. Ledig, L. Theis, Ferenc Huszár, J. Caballero, Andrew Aitken, Alykhan Tejani, J. Totz, Zehan Wang, and W. Shi. 2017. Photo-realistic single image super-resolution using a generative adversarial network. In Proceedings of the IEEE/CVF Conference on Computer Vision and Pattern Recognition (CVPR'17).

[89] J. Lee, J. Lee, and H. J. Yoo. 2020. SRNPU: An energy-efficient CNN-based super-resolution processor with tile-based selective super-resolution in mobile devices. IEEE f. Emerg. Select. Topics Circ. Syst. 10, 3 (2020), 320-334.

[90] Kyungjin Lee, Juheon Yi, Youngki Lee, Sunghyun Choi, and Young Min Kim. 2020. GROOT: A real-time streaming system of high-fidelity volumetric videos. In Proceedings of the 26th International Conference on Mobile Computing and Networking (MobiCom'20).

[91] Royson Lee, L. Dudziak, M. Abdelfattah, Stylianos I. Venieris, H. Kim, Hongkai Wen, and N. Lane. 2020. Journey towards tiny perceptual super-resolution. In Proceedings of the European Conference on Computer Vision (ECCV'20). 
[92] Royson Lee, Stylianos I. Venieris, L. Dudziak, S. Bhattacharya, and N. Lane. 2019. MobiSR: Efficient on-device superresolution through heterogeneous mobile processors. In Proceedings of the 25th International Conference on Mobile Computing and Networking (MobiCom'19).

[93] Huixia Li, Chenqian Yan, Shaohui Lin, Xiawu Zheng, B. Zhang, Fan Yang, and Rongrong Ji. 2020. PAMS: Quantized super-resolution via parameterized max scale. In Proceedings of the European Conference on Computer Vision.

[94] X. Li, M. A. Salehi, M. Bayoumi, N. Tzeng, and R. Buyya. 2018. Cost-efficient and robust on-demand video transcoding using heterogeneous cloud services. IEEE Trans. Parallel Distrib. Syst. 29, 3 (2018), 556-571.

[95] Y. Li, D. Liu, H. Li, Lianghuan Li, Z. Li, and F. Wu. 2019. Learning a convolutional neural network for image compactresolution. IEEE Trans. Image Process. 28, 3 (2019), 1092-1107.

[96] Yule Li, J. Shi, and D. Lin. 2018. Low-latency video semantic segmentation.In Proceedings of the IEEE/CVF Conference on Computer Vision and Pattern Recognition (CVPR'18).

[97] Yuheng Li, Yiping Zhang, and Ruixi Yuan. 2011. Measurement and analysis of a large scale commercial mobile internet TV system. In Proceedings of the ACM SIGCOMM Conference on Internet Measurement Conference (IMC'11). 209-224.

[98] Z. Li, M. A. Kaafar, K. Salamatian, and G. Xie. 2017. Characterizing and modeling user behavior in a large-scale mobile live streaming system. IEEE Trans. Circ. Syst. Vid. Technol. 27, 12 (2017), 2675-2686.

[99] Zhenyu Li, Jiali Lin, Marc-Ismael Akodjenou, Gaogang Xie, Mohamed Ali Kaafar, Yun Jin, and Gang Peng. 2012. Watching videos from everywhere: A study of the PPTV mobile VoD system. In Proceedings of the Internet Measurement Conference (IMC'12). 185-198.

[100] Z. Li, X. Zhu, J. Gahm, R. Pan, H. Hu, A. C. Begen, and D. Oran. 2014. Probe and adapt: Rate adaptation for HTTP video streaming at scale. IEEE f. Select. Areas Commun. 32, 4 (2014), 719-733.

[101] Li-Shen Juhn and Li-Ming Tseng. 1997. Harmonic broadcasting for video-on-demand service. IEEE Trans. Broadcast. 43, 3 (1997), 268-271.

[102] Renjie Liao, X. Tao, R. Li, Z. Ma, and J. Jia. 2015. Video super-resolution via deep draft-ensemble learning.In Proceedings of the IEEE International Conference on Computer Vision (ICCV'15).

[103] Melissa Licciardello, Maximilian Grüner, and Ankit Singla. 2020. Understanding video streaming algorithms in the wild. In Passive and Active Measurement (PAM), Anna Sperotto, Alberto Dainotti, and Burkhard Stiller (Eds.). Springer International Publishing, Cham, 298-313.

[104] Bee Lim, Sanghyun Son, Heewon Kim, Seungjun Nah, and Kyoung Mu Lee. 2017. Enhanced deep residual networks for single image super-resolution. In Proceedings of the IEEE/CVF Conference on Computer Vision and Pattern Recognition Workshops (CVPRW'17).

[105] H. Liu, Zhubo Ruan, Peng Zhao, F. Shang, Linlin Yang, and Yuanyuan Liu. 2020. Video super resolution based on deep learning: A comprehensive survey. ArXiv abs/2007.12928 (2020).

[106] Luyang Liu, Hongyu Li, and Marco Gruteser. 2019. Edge assisted real-time object detection for mobile augmented reality. In Proceedings of the 25th International Conference on Mobile Computing and Networking (MobiCom'19).

[107] Luyang Liu, Ruiguang Zhong, Wuyang Zhang, Yunxin Liu, Jiansong Zhang, Lintao Zhang, and Marco Gruteser. 2018. Cutting the cord: Designing a high-quality untethered VR system with low latency remote rendering. In Proceedings of the 16th International Conference on Mobile Systems, Applications, and Services (MobiSys'18). 68-80.

[108] Xin Liu, Yuang Li, Josh Fromm, Yuntao Wang, Ziheng Jiang, Alex Mariakakis, and Shwetak Patel. 2021. SplitSR: An end-to-end approach to super-resolution on mobile devices. Proc. ACM Interact. Mob. Wear. Ubiq. Technol. 5, 1 (2021), 20 pages.

[109] Wen-Chih Lo, Ching-Ling Fan, Jean Lee, Chun-Ying Huang, Kuan-Ta Chen, and Cheng-Hsin Hsu. 2017. $360^{\circ}$ video viewing dataset in head-mounted virtual reality. In Proceedings of the 8th ACM on Multimedia Systems Conference (MMSys'17). 211-216.

[110] Jonathan Long, Evan Shelhamer, and Trevor Darrell. 2015. Fully convolutional networks for semantic segmentation. In Proceedings of the IEEE Conference on Computer Vision and Pattern Recognition (CVPR'15). 3431-3440.

[111] Cheng Ma, Yongming Rao, Yean Cheng, Ce Chen, Jiwen Lu, and J. Zhou. 2020. Structure-preserving super resolution with gradient guidance. In Proceedings of the IEEE/CVF Conference on Computer Vision and Pattern Recognition (CVPR'20).

[112] Ningning Ma, X. Zhang, Hai-Tao Zheng, and Jian Sun. 2018. ShuffleNet V2: Practical guidelines for efficient CNN architecture design. In Proceedings of the European Conference on Computer Vision (ECCV'18).

[113] Y. Ma, Hongyu Xiong, Zhe Hu, and L. Ma. 2019. Efficient super resolution using binarized neural network.In Proceedings of the IEEE/CVF Conference on Computer Vision and Pattern Recognition Workshops (CVPRW'19).

[114] Hongzi Mao, R. Netravali, and M. Alizadeh. 2017. Neural adaptive video streaming with pensieve.In Proceedings of the SIGCOMM Conference.

[115] D. Martin, C. Fowlkes, D. Tal, and J. Malik. 2001. A database of human segmented natural images and its application to evaluating segmentation algorithms and measuring ecological statistics. In Proceedings of the IEEE International Conference on Computer Vision (ICCV'01). 
[116] Roey Mechrez, I. Talmi, Firas Shama, and L. Zelnik-Manor. 2018. Maintaining natural image statistics with the contextual loss. In Proceedings of the Asian Conference on Computer Vision.

[117] Anish Mittal, Rajiv Soundararajan, and Alan C. Bovik. 2013. Making a "completely blind" image quality analyzer. IEEE Sig. Process. Lett. 20, 3 (2013), 209-212.

[118] R. K. P. Mok, E. W. W. Chan, and R. K. C. Chang. 2011. Measuring the quality of experience of HTTP video streaming. In Proceedings of the 12th IFIP/IEEE International Symposium on Integrated Network Management (IM'11) and Workshops. 485-492.

[119] Vinod Nair and Geoffrey E. Hinton. 2010. Rectified linear units improve restricted Boltzmann machines. In Proceedings of the 27th International Conference on International Conference on Machine Learning (ICML'10). Omnipress, 807-814.

[120] NVIDIA. 2020. NVIDIA Maxine - Cloud-AI Video-Streaming Platform. Retrieved from https://developer.nvidia.com/ maxine.

[121] Seobin Park, Jinsu Yoo, Donghyeon Cho, Jiwon Kim, and Tae Hyun Kim. 2020. Fast adaptation to super-resolution networks via meta-learning. In Proceedings of the European Conference on Computer Vision (ECCV'20).

[122] K. Piamrat, C. Viho, J. Bonnin, and A. Ksentini. 2009. Quality of experience measurements for video streaming over wireless networks. In Proceedings of the 6th International Conference on Information Technology: New Generations (ITNG'09). 1184-1189.

[123] F. Qian, B. Han, Qingyang Xiao, and V. Gopalakrishnan. 2018. Flare: Practical viewport-adaptive 360-degree video streaming for mobile devices.In Proceedings of the 24th International Conference on Mobile Computing and Networking (MobiCom'18).

[124] Parthasarathy Ranganathan, Daniel Stodolsky, Jeff Calow, Jeremy Dorfman, Marisabel Guevara, Clinton Wills Smullen IV, Aki Kuusela, Raghu Balasubramanian, Sandeep Bhatia, Prakash Chauhan, Anna Cheung, In Suk Chong, Niranjani Dasharathi, Jia Feng, Brian Fosco, Samuel Foss, Ben Gelb, Sara J. Gwin, Yoshiaki Hase, Da-ke He, C. Richard Ho, Roy W. Huffman Jr., Elisha Indupalli, Indira Jayaram, Poonacha Kongetira, Cho Mon Kyaw, Aaron Laursen, Yuan Li, and Fong Lou. 2021. Warehouse-scale video acceleration: Co-design and deployment in the wild. In Proceedings of the 26th ACM International Conference on Architectural Support for Programming Languages and Operating Systems (ASPLOS'21). 600-615.

[125] A. Ranjan and Michael J. Black. 2017. Optical flow estimation using a spatial pyramid network.In Proceedings of the IEEE Conference on Computer Vision and Pattern Recognition (CVPR'17).

[126] Leonid I. Rudin, Stanley Osher, and Emad Fatemi. 1992. Nonlinear total variation based noise removal algorithms. Phys. D 60, 1-4 (Nov. 1992), 259-268.

[127] Mehdi S. M. Sajjadi, Raviteja Vemulapalli, and M. Brown. 2018. Frame-recurrent video super-resolution.In Proceedings of the IEEE/CVF Conference on Computer Vision and Pattern Recognition.

[128] Haichen Shen, Seungyeop Han, Matthai Philipose, and Arvind Krishnamurthy. 2017. Fast video classification via adaptive cascading of deep models. In Proceedings of the IEEE Conference on Computer Vision and Pattern Recognition (CVPR'17).

[129] W. Shi, J. Caballero, Ferenc Huszár, J. Totz, A. Aitken, R. Bishop, D. Rueckert, and Zehan Wang. 2016. Real-time single image and video super-resolution using an efficient sub-pixel convolutional neural network.In Proceedings of the IEEE Conference on Computer Vision and Pattern Recognition (CVPR'16).

[130] Assaf Shocher, N. Cohen, and M. Irani. 2018. "Zero-shot" super-resolution using deep internal learning. In Proceedings of the IEEE/CVF Conference on Computer Vision and Pattern Recognition (CVPR'18).

[131] Laurent Sifre and Stéphane Mallat. 2014. Rigid-motion scattering for image classification author. English. Supervisor: Prof. Stéphane Mallat. Ph. D. Thesis. Ecole Polytechnique.

[132] Jae Woong Soh, Sunwoo Cho, and N. I. Cho. 2020. Meta-transfer learning for zero-shot super-resolution.In Proceedings of the IEEE/CVF Conference on Computer Vision and Pattern Recognition (CVPR'20).

[133] Jae Woong Soh, G. Y. Park, Junho Jo, and N. I. Cho. 2019. Natural and realistic single image super-resolution with explicit natural manifold discrimination. In Proceedings of the IEEE/CVF Conference on Computer Vision and Pattern Recognition (CVPR'19).

[134] Dehua Song, Chang Xu, Xu Jia, Yiyi Chen, Chunjing Xu, and Yunhe Wang. 2020. Efficient residual dense block search for image super-resolution. In Proceedings of the Association for the Advancement of Artificial Intelligence Conference (AAAI'20).

[135] J. Song, Y. Cho, J. Park, J. Jang, S. Lee, J. Song, J. Lee, and I. Kang. 2019. 7.1 An 11.5TOPS/W 1024-MAC butterfly structure dual-core sparsity-aware neural processing unit in $8 \mathrm{~nm}$ flagship mobile SoC. In Proceedings of the IEEE International Solid-State Circuits Conference (ISSCC'19). 130-132.

[136] Kevin Spiteri, Ramesh Sitaraman, and Daniel Sparacio. 2018. From theory to practice: Improving bitrate adaptation in the DASH reference player. In Proceedings of the 9th ACM Multimedia Systems Conference (MMSys'18). $123-137$. 
[137] K. Spiteri, R. Urgaonkar, and R. K. Sitaraman. 2016. BOLA: Near-optimal bitrate adaptation for online videos. In Proceedings of the 35th IEEE International Conference on Computer Communications. 1-9.

[138] Thomas Stockhammer. 2011. Dynamic adaptive streaming over HTTP -: Standards and design principles. In Proceedings of the 2nd ACM Conference on Multimedia Systems (MMSys'11). 133-144.

[139] Deqing Sun, X. Yang, Ming-Yu Liu, and J. Kautz. 2018. PWC-Net: CNNs for optical flow using pyramid, warping, and cost volume. In Proceedings of the IEEE/CVF Conference on Computer Vision and Pattern Recognition (CVPR'18).

[140] Yi Sun, Xiaoqi Yin, Junchen Jiang, Vyas Sekar, Fuyuan Lin, Nanshu Wang, Tao Liu, and Bruno Sinopoli. 2016. CS2P: Improving video bitrate selection and adaptation with data-driven throughput prediction. In Proceedings of the ACM SIGCOMM Conference (SIGCOMM'16). 272-285.

[141] X. Tao, H. Gao, Renjie Liao, J. Wang, and J. Jia. 2017. Detail-revealing deep video super-resolution.In Proceedings of the IEEE International Conference on Computer Vision (ICCV'17).

[142] Surat Teerapittayanon, Bradley McDanel, and H. T. Kung. 2016. BranchyNet: Fast inference via early exiting from deep neural networks. In Proceedings of the 23rd International Conference on Pattern Recognition (ICPR'16). 2464-2469.

[143] Yapeng Tian, Yulun Zhang, Yun Fu, and Chenliang Xu. 2020. TDAN: Temporally-deformable alignment network for video super-resolution.In Proceedings of the IEEE/CVF Conference on Computer Vision and Pattern Recognition (CVPR'20).

[144] R. Timofte, Eirikur Agustsson, L. Gool, M. Yang, Lei Zhang, Bee Lim, Sanghyun Son, Heewon Kim, Seungjun Nah, K. Lee, Xintao Wang, Yapeng Tian, K. Yu, Yulun Zhang, Shixiang Wu, C. Dong, L. Lin, Y. Qiao, C. C. Loy, W. Bae, Jae Jun Yoo, Yoseob Han, J. C. Ye, Jae-Seok Choi, M. Kim, Yuchen Fan, J. Yu, Wei Han, Ding Liu, Haichao Yu, Zhangyang Wang, Humphrey Shi, X. Wang, T. Huang, Yunjin Chen, Kai Zhang, W. Zuo, Z. Tang, Linkai Luo, S. Li, M. Fu, L. Cao, Wen Heng, G. Bui,Truc Le, Ye Duan, D. Tao, Ruxin Wang, Xu Lin, Jianxin Pang, Jinchang Xu, Y. Zhao, Xiangyu Xu, Jin shan Pan, Deqing Sun, Y. Zhang, X. Song, Yuchao Dai, X. Qin, X. Huynh, Tiantong Guo, H. Mousavi, T. Vu, V. Monga, C. Cruz, K. Egiazarian, V. Katkovnik, Rakesh Mehta, A. Jain, Abhinav Agarwalla, Ch V., Sai Praveen, Ruofan Zhou, Hongdiao Wen, C. Zhu, Zhiqiang Xia, Z. Wang, and Q. Guo. 2017. NTIRE 2017 challenge on single image super-resolution: Methods and results.In Proceedings of the IEEE Conference on Computer Vision and Pattern Recognition Workshops (CVPRW'17).

[145] S. I. Venieris and C. S. Bouganis. 2019. fpgaConvNet: Mapping regular and irregular convolutional neural networks on FPGAs. IEEE Trans. Neural Netw. Learn. Syst. 30, 2 (2019), 326-342.

[146] Stylianos I. Venieris, Alexandros Kouris, and Christos-Savvas Bouganis. 2018. Deploying deep neural networks in the embedded space. In Proceedings of the 2nd International Workshop on Embedded and Mobile Deep Learning (EMDL'18).

[147] Stylianos I. Venieris, Alexandros Kouris, and Christos-Savvas Bouganis. 2018. Toolflows for mapping convolutional neural networks on FPGAs: A survey and future directions. ACM Comput. Surv. 51, 3 (June 2018). DOI : https://doi. org $/ 10.1145 / 3186332$

[148] Xintao Wang, Kelvin C. K. Chan, K. Yu, C. Dong, and Chen Change Loy. 2019. EDVR: Video restoration with enhanced deformable convolutional networks.In Proceedings of the IEEE/CVF Conference on Computer Vision and Pattern Recognition Workshops (CVPRW'19).

[149] Xin Wang, Fisher Yu, Zi-Yi Dou, Trevor Darrell, and Joseph E. Gonzalez. 2018. SkipNet: Learning dynamic routing in convolutional networks. In Proceedings of the European Conference on Computer Vision (ECCV'18). 409-424.

[150] Xintao Wang, K. Yu, C. Dong, X. Tang, and Chen Change Loy. 2019. Deep network interpolation for continuous imagery effect transition. In Proceedings of the IEEE/CVF Conference on Computer Vision and Pattern Recognition (CVPR'19).

[151] Xintao Wang, Ke Yu, Shixiang Wu, Jinjin Gu, Yihao Liu, Chao Dong, Chen Change Loy, Yu Qiao, and Xiaoou Tang. 2018. ESRGAN: Enhanced super-resolution generative adversarial networks. In Proceedings of the European Conference on Computer Vision Workshops (ECCVW'18).

[152] Yiding Wang, Weiyan Wang, Junxue Zhang, J. Jiang, and K. Chen. 2019. Bridging the edge-cloud barrier for real-time advanced vision analytics. In Proceedings of the HotCloud Conference.

[153] Yulong Wang, Xiaolu Zhang, Xiaolin Hu, Bo Zhang, and Hang Su. 2020. Dynamic network pruning with interpretable layerwise channel selection. In Proceedings of the AAAI Conference. 6299-6306.

[154] Zizhao Wang, Wei Bao, Dong Yuan, Liming Ge, Nguyen H. Tran, and Albert Y. Zomaya. 2019. SEE: Scheduling early exit for mobile DNN inference during service outage. In Proceedings of the 22nd International ACM Conference on Modeling, Analysis and Simulation of Wireless and Mobile Systems (MSWIM'19). 279-288.

[155] Zhou Wang, Alan C. Bovik, Hamid R. Sheikh, and Eero P. Simoncelli. 2004. Image quality assessment: from error visibility to structural similarity. IEEE Trans. Image Process. 13, 4 (2004), 600-612.

[156] Zhihao Wang, Jian Chen, and S. Hoi. 2020. Deep learning for image super-resolution: A survey. IEEE Trans. Pattern Anal. Mach. Intell. (2020). DOI : 10.1109/TPAMI.2020.2982166

[157] C. Wu, D. Brooks, K. Chen, D. Chen, S. Choudhury, M. Dukhan, K. Hazelwood, E. Isaac, Y. Jia, B. Jia, T. Leyvand, H. Lu, Y. Lu, L. Qiao, B. Reagen, J. Spisak, F. Sun, A. Tulloch, P. Vajda, X. Wang, Y. Wang, B. Wasti, Y. Wu, R. Xian, S. 
Yoo, and P. Zhang. 2019. Machine learning at Facebook: Understanding inference at the edge. In Proceedings of the IEEE International Symposium on High Performance Computer Architecture (HPCA'19). 331-344.

[158] X. Xiang, Yapeng Tian, Yulun Zhang, Y. Fu, J. Allebach, and Chenliang Xu. 2020. Zooming slow-mo: Fast and accurate one-stage space-time video super-resolution.In Proceedings of the IEEE/CVF Conference on Computer Vision and Pattern Recognition (CVPR'20).

[159] Mingqing Xiao, Shuxin Zheng, Chang Liu, Yaolong Wang, Di He, Guolin Ke, Jiang Bian, Zhouchen Lin, and Tie-Yan Liu. 2020. Invertible image rescaling. In Proceedings of the European Conference on Computer Vision (ECCV'20).

[160] Jingwei Xin, Nannan Wang, Xinrui Jiang, Jie Li, Heng Huang, and Xinbo Gao. 2020. Binarized neural network for single image super resolution. In Proceedings of the European Conference on Computer Vision (ECCV'20).

[161] Qunliang Xing, Mai Xu, Tianyi Li, and Zhenyu Guan. 2020. Early exit or not: Resource-efficient blind quality enhancement for compressed images. In Proceedings of the European Conference on Computer Vision (ECCV'20). Springer.

[162] Jianchao Yang, John Wright, Thomas S. Huang, and Yi Ma. 2010. Image super-resolution via sparse representation. IEEE Trans. Image Process. 19, 11 (2010), 2861-2873.

[163] Wenming Yang, X. Zhang, Yapeng Tian, W. Wang, Jing-Hao Xue, and Qingmin Liao. 2019. Deep learning for single image super-resolution: A brief review. IEEE Trans. Multimedia 21, 12 (2019), 3106-3121.

[164] Hyunho Yeo, Chan Ju Chong, Youngmok Jung, Juncheol Ye, and Dongsu Han. 2020. NEMO: Enabling neuralenhanced video streaming on commodity mobile devices. In Proceedings of the 26th International Conference on Mobile Computing and Networking (MobiCom'20).

[165] H. Yeo, Sunghyun Do, and D. Han. 2017. How will deep learning change internet video delivery? In Proceedings of the HotNets Conference.

[166] H. Yeo, Youngmok Jung, Jaehong Kim, Jinwoo Shin, and D. Han. 2018. Neural adaptive content-aware internet video delivery. In Proceedings of the 13th USENIX Symposium on Operating Systems Design and Implementation (OSDI'18).

[167] Junheon Yi, Seongwon Kim, Joongheon Kim, and Sunghyun Choi. 2020. Supremo: Cloud-assisted low-latency superresolution in mobile devices. IEEE Trans. Mob. Comput. (2020). DOI : 10.1109/TMC.2020.3025300

[168] Xiaoqi Yin, A. Jindal, V. Sekar, and B. Sinopoli. 2015. A control-theoretic approach for dynamic adaptive video streaming over HTTP. In Proceedings of the SIGCOMM Conference.

[169] Hongliang Yu, Dongdong Zheng, Ben Y. Zhao, and Weimin Zheng. 2006. Understanding user behavior in large-scale video-on-demand systems. In Proceedings of the 1st ACM SIGOPS/EuroSys European Conference on Computer Systems (EuroSys'06). 333-344.

[170] E. Zakharov, Aleksei Ivakhnenko, Aliaksandra Shysheya, and V. Lempitsky. 2020. Fast bi-layer neural synthesis of one-shot realistic head avatars. In Proceedings of the European Conference on Computer Vision (ECCV'20).

[171] Haoyu Zhang, Ganesh Ananthanarayanan, Peter Bodik, Matthai Philipose, Paramvir Bahl, and Michael J. Freedman. 2017. Live video analytics at scale with approximation and delay-tolerance. In Proceedings of the 14th USENIX Conference on Networked Systems Design and Implementation (NSDI'17). 377-392.

[172] Huanhuan Zhang, Anfu Zhou, Jiamin Lu, Ruoxuan Ma, Yuhan Hu, Cong Li, Xinyu Zhang, Huadong Ma, and Xiaojiang Chen. 2020. OnRL: Improving mobile video telephony via online reinforcement learning. In Proceedings of the 26th International Conference on Mobile Computing and Networking (MobiCom'20).

[173] Linfeng Zhang, Zhanhong Tan, Jiebo Song, Jingwei Chen, Chenglong Bao, and Kaisheng Ma. 2019. SCAN: A scalable neural networks framework towards compact and efficient models. In Proceedings of the International Conference on Advances in Neural Information Processing Systems (NeurIPS'19). 4027-4036.

[174] Richard Zhang, Phillip Isola, Alexei A. Efros, Eli Shechtman, and Oliver Wang. 2018. The unreasonable effectiveness of deep features as a perceptual metric. In Proceedings of the IEEE/CVF Conference on Computer Vision and Pattern Recognition (CVPR'18).

[175] Yulun Zhang, Kunpeng Li, Kai Li, Lichen Wang, Bineng Zhong, and Yun Fu. 2018. Image super-resolution using very deep residual channel attention networks. In Proceedings of the European Conference on Computer Vision (ECCV'18).

[176] Hengshuang Zhao, Xiaojuan Qi, Xiaoyong Shen, J. Shi, and J. Jia. 2018. ICNet for real-time semantic segmentation on high-resolution images. In Proceedings of the European Conference on Computer Vision (ECCV'18).

[177] C. Zhu, X. Lin, and Lap-Pui Chau. 2002. Hexagon-based search pattern for fast block motion estimation. IEEE Trans. Circ. Syst. Vid. Technol. 12, 5 (2002), 349-355.

[178] X. Zhu, H. Hu, Stephen Lin, and Jifeng Dai. 2019. Deformable ConvNets V2: More deformable, better results.In Proceedings of the IEEE/CVF Conference on Computer Vision and Pattern Recognition (CVPR'19).

[179] Barret Zoph and Quoc V. Le. 2017. Neural architecture search with reinforcement learning. In Proceedings of the International Conference on Learning Representations (ICLR'17).

[180] Liad Pollak Zuckerman, S. Bagon, Eyal Naor, George Pisha, and M. Irani. 2020. Across scales \& across dimensions: Temporal super-resolution using deep internal learning. In Proceedings of the European Conference on Computer Vision (ECCV'20).

Received October 2020; revised May 2021; accepted May 2021 